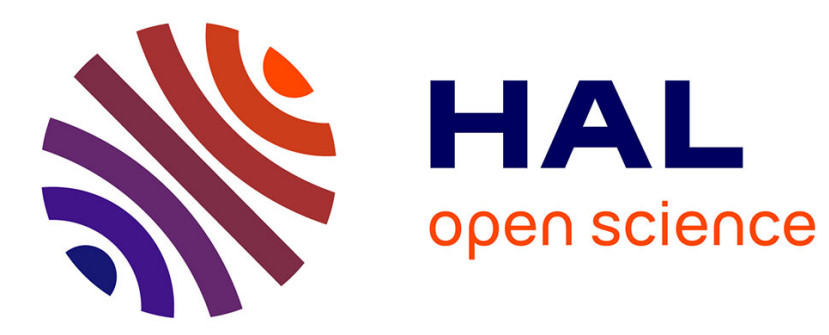

\title{
Analytic model of a plane resistive magnetohydrodynamic shock with Hall effect
}

Roland P. H. Berton

\section{To cite this version:}

Roland P. H. Berton. Analytic model of a plane resistive magnetohydrodynamic shock with Hall effect. CEAS Space Journal, 2021, 13 (1), pp.83-110. 10.1007/s12567-020-00303-x . hal-03183996

\section{HAL Id: hal-03183996 \\ https://hal.science/hal-03183996}

Submitted on 29 Mar 2021

HAL is a multi-disciplinary open access archive for the deposit and dissemination of scientific research documents, whether they are published or not. The documents may come from teaching and research institutions in France or abroad, or from public or private research centers.
L'archive ouverte pluridisciplinaire HAL, est destinée au dépôt et à la diffusion de documents scientifiques de niveau recherche, publiés ou non, émanant des établissements d'enseignement et de recherche français ou étrangers, des laboratoires publics ou privés. 


\title{
Analytic model of a plane resistive magnetohydrodynamic shock with Hall effect
}

Roland P.H. Berton ${ }^{1}$

\begin{abstract}
In the present work, our formerly published analytical model of resistive magnetohydrodynamic (MHD) shock without Hall effect has been extended to include the Hall effect. Original jump conditions at a plane oblique shock, analogous to the Rankine-Hugoniot formulas, with a moderately resistive air plasma downstream are derived. Viscous and Ohmic dissipations are neglected, but the jump of isentropic exponent, caused by molecular dissociations behind the shock, is also included in the model. Then, for an application to atmospheric entry problems, a shock fitting procedure with realistic geometrical configurations and ambient conditions at two altitudes is worked out by the coupling of these MHD jumps with thermodynamic correlations at equilibrium and an electrical conductivity model. Fundamental features put into evidence are the reduction and saturation of the MHD interaction by the Hall effect as the magnetic field increases, and the crucial role played by the ion slip factor in the phenomenon. Paradoxically, the Hall effect arises because of the dominance of the magnetic field over collisions, and eventually it counters the MHD interaction. The validity of the assumption of plasma equilibrium and possible validation experiments for the flight conditions considered are also discussed.
\end{abstract}

Keywords : Magnetohydrodynamics $\cdot$ Plasma $\cdot$ Shock waves

\section{Introduction}

The entry of a space vehicle into a planetary atmosphere at a hypersonic speed raises many interesting questions relevant of aerodynamics, plasma physics, radio transmission, and material erosion. Thus, a lot of works have been carried on in the past fifty years about the magnetohydrodynamic (MHD) problems resulting from the application of a magnetic field for flow control [5][21], radio mitigation [30], or heat reduction [6][19][35][36].

In a previous work, we worked out an analytic model of resistive MHD shock without Hall effect, in order to focus on the jumps due to the finite conductivity of the plasma, and to the real gas effects caused by molecular dissociations [3]. Here, we shall add the contribution of the Hall effect for a plane MHD oblique shock, and develop in detail the basic formalism, about which we recently presented results without mathematical proofs [4].

Owing to the advantage of fast convergence, shock-capturing procedures are widely used in computational fluid dynamics (CFD), but they have the drawback of lesser accuracy [38]. Conversely, fitting procedures are more accurate, but they are also more time-consuming [33][34][43]. We recall that the motivation of our approach is to provide CFD shock-fitting methods with jump relations involving real gas effects (due to molecular dissociation) and MHD effects (due to action of a magnetic field on the shock-generated plasma), as an alternative to the original Rankine-Hugoniot giving the pressure and mass density ratios [28][42]:

$$
\frac{p_{2}}{p_{1}}=\frac{2 \gamma M_{1}^{2} \cos ^{2} \chi_{1}-(\gamma-1)}{\gamma+1}, \quad \frac{\rho_{2}}{\rho_{1}}=\frac{(\gamma+1) M_{1}^{2} \cos ^{2} \chi_{1}}{(\gamma-1) M_{1}^{2} \cos ^{2} \chi_{1}+2},
$$

for an ideal gas, without gas decomposition (no jump of isentropic exponent $\gamma$ ), and for a pure hydrodynamic (HD) flow (no applied magnetic field).

So far, no theory has been proposed to examine analytically the behavior of a plane resistive shock subject to an externally applied magnetic field with a developed Hall effect, at small magnetic Reynolds number $\left(R_{m} \approx 10^{-3}\right)$. Actually, a considerable amount of research has been devoted to MHD shocks at high magnetic Reynolds number $\left(R_{m} \geq 10^{3}\right)$ in a geophysical or astrophysical background [26]. More recently, Hall MHD has become a part of astrophysical magnetohydrodynamics in its own [22], because the Hall effect is likely to develop in most of magnetized celestial bodies, and to impact MHD processes, including turbulence [27], dynamo [29], star formation [7] and magnetic reconnection [15]. The main difference between both situations is that, as pointed out in our previous work by considering the magnetic Reynolds number [3], the magnetic field does not evolve in our problem, while it is strongly flow-dependent in geophysical and astrophysical situations.

\footnotetext{
${ }^{1}$ DOTA, ONERA, Université Paris Saclay, F-91123 Palaiseau - France roland.berton@onera.fr
} 
The jump equations will be first derived in Sect. 2 in two steps, first by integration of differential equations (Sect. 2.2), and second by solving a nonlinear algebraic system (Sect. 2.3). Thus, the pressure and mass density ratios corresponding to relations (1) will be expressed as solutions of quadratic equations. Then, the plane-shock fitting procedure with Hall effect will be described and illustrated in Sect. 3. In particular, two geometric configurations will be chosen, in order to display the influence of the Hall effect on the shock properties, as the incident Mach number and the magnetic field strength are varied.

The nondimensional key parameter currently used to characterize the MHD interaction, known as interaction parameter, or Stuart number, is difficult to estimate in certain situations without considering the differential equations leading to its definition. Otherwise, the classical order-of-magnitude definition may be misleading and confusing. This is an important problem that will be examined in the discussion of Sect. 3.3. In Sect. 3.4, we examine the possibilities of experimental validation, the validity of thermochemical equilibrium, and the pertinence of the power-law conductivity model.

Notations used throughout the paper are defined in Table 1. For any given quantity, the subscripts 1 and 2 refer respectively to the upstream and downstream values. For any variable $X,[X]$ denotes the difference $X_{2}-X_{1}$, that we shall hereafter call "jump".

\section{Jump relations}

\subsection{Basic differential equations}

The configuration considered for the derivation of jumps is that of a plane oblique shock, inclined at an angle $\chi_{1}$ with respect to the mean upstream flow moving at the relative velocity $\boldsymbol{V}_{1}$, to which is applied a magnetic field $\boldsymbol{B}$ (Fig. 1). $\boldsymbol{V}_{2}$ ' is the projection of $\boldsymbol{V}_{2}$ in the $\left(\boldsymbol{V}_{1}, \boldsymbol{B}\right)$ plane according to:

$V_{2}=u_{2}+v_{2}+w_{2}=V_{2}^{\prime}+w_{2}$.

The basic equations of magnetohydrodynamics are composed of the Navier-Stokes and Maxwell's equations complemented with Ohm's law. The $y$ and $z$ axes are supposed to be directions of invariance. Therefore the electric field components $E_{y}$ and $E_{z}$, being respectively the $y$ and $z$ derivatives of the electric potential, vanish $\left(E_{y}=0 ; E_{z}=0\right)$. Under these simplifications the governing equations are written here as [46]:

$$
\left\{\begin{array}{l}
\frac{\mathrm{d}}{\mathrm{d} x}(\rho u)=0 \\
\rho u \frac{\mathrm{d} u}{\mathrm{~d} x}=-\frac{\mathrm{d} p}{\mathrm{~d} x}+\frac{\mathrm{d}}{\mathrm{d} x}\left(\frac{4}{3} \mu \frac{\mathrm{d} u}{\mathrm{~d} x}\right)-\left\{\sigma_{P}\left(u \sin \chi_{b}-v \cos \chi_{b}\right) B-\sigma_{H}\left(E_{x} \sin \chi_{b}-w B\right)\right\} B \sin \chi_{b} \\
\rho u \frac{\mathrm{d} v}{\mathrm{~d} x}=\frac{\mathrm{d}}{\mathrm{d} x}\left(\frac{4}{3} \mu \frac{\mathrm{d} v}{\mathrm{~d} x}\right)+\left\{\sigma_{P}\left(u \sin \chi_{b}-v \cos \chi_{b}\right) B-\sigma_{H}\left(E_{x} \sin \chi_{b}-w B\right)\right\} B \cos \chi_{b} \\
\rho u \frac{\mathrm{d} w}{\mathrm{~d} x}=\frac{\mathrm{d}}{\mathrm{d} x}\left(\frac{4}{3} \mu \frac{\mathrm{d} w}{\mathrm{~d} x}\right)+\sigma_{H}\left(u \sin \chi_{b}-v \cos \chi_{b}\right) B^{2}+\sigma_{P}\left(E_{x} \sin \chi_{b}-w B\right) B \\
\rho u \frac{\mathrm{d}}{\mathrm{d} x}\left(\frac{\gamma}{\gamma-1} \frac{p}{\rho}+\frac{u^{2}+v^{2}+w^{2}}{2}\right)=\frac{\mathrm{d}}{\mathrm{d} x}\left(\frac{4}{3} \mu u \frac{\mathrm{d} u}{\mathrm{~d} x}+\frac{4}{3} \mu v \frac{\mathrm{d} v}{\mathrm{~d} x}+\frac{4}{3} \mu w \frac{\mathrm{d} w}{\mathrm{~d} x}\right)+j_{x} E_{x}
\end{array}\right.
$$

This set of equations is complemented with Ohm's law, making it possible to express the electric current density as follows, when electronic pressure gradients are neglected [46]:

$$
\left\{\begin{array}{l}
J_{x}=\left(\sigma_{H} \cos ^{2} \chi_{b}+\sigma_{P} \sin ^{2} \chi_{b}\right) E_{x}+\sigma_{H}\left(u \sin \chi_{b}-v \cos \chi_{b}\right) B \sin \chi_{b}-\sigma_{P} w B \sin \chi_{b} \\
J_{y}=\left(\sigma_{e}-\sigma_{P}\right) E_{x} \sin \chi_{b} \cos \chi_{b}-\sigma_{H}\left(u \sin \chi_{b}-v \cos \chi_{b}\right) B \cos \chi_{b}+\sigma_{P} w B \cos \chi_{b} \\
J_{z}=\sigma_{P}\left(u \sin \chi_{b}-v \cos \chi_{b}\right) B-\sigma_{H}\left(E_{x} \sin \chi_{b}-w B\right)
\end{array}\right.
$$

In these equations, $\sigma_{e}, \sigma_{P}$ and $\sigma_{H}$ denote respectively the ordinary, Pedersen and Hall electrical conductivities, that can be written, for a weakly ionized plasma [46]:

$$
\sigma_{e}=\frac{n_{e} q_{e}^{2}}{m_{e} v_{e}} \quad \sigma_{P}=\sigma_{e} \frac{1+s}{(1+s)^{2}+\beta_{e}^{2}} \quad \sigma_{H}=\sigma_{e} \frac{\beta_{e}}{(1+s)^{2}+\beta_{e}^{2}} .
$$

They involve the Hall parameters $\beta_{e}$ and $\beta_{i}$ for electrons and ions respectively, such that:

$$
\beta_{e}=\frac{q_{e} B}{m_{e} v_{e}}, \quad \beta_{i}=\frac{q_{i} B}{m_{i} v_{i}},
$$


and the associated slip factor $s$ :

$s=\beta_{e} \beta_{i}$.

In addition, following other authors [36][39], in order to be consistent with our previous approach [3], we assume a power-law relationship between the ordinary electrical conductivity $\sigma_{e}$ and the temperature $T$ :

$\sigma_{e}=\sigma_{0}\left(\frac{T}{T_{0}}\right)^{\alpha}$

with the constants: $\alpha=2, T_{0}=8000 \mathrm{~K}, \sigma_{0}=731 \mathrm{~S} \mathrm{~m}^{-1}$.

The equation of conservation of magnetic flux $(\nabla \cdot \boldsymbol{B}=0)$, and the fact that the magnetic Reynolds number is small $\left(\mu_{0}|\boldsymbol{J}| \ll|\nabla \times \boldsymbol{B}|\right)$ show that the applied magnetic field remains practically unaltered by the fluid flowing across the shock. Therefore, its magnitude $B$ and angle $\chi_{b}$ will be kept constant in the following sections.

The equation of conservation of electric charge $(\nabla \cdot J=0)$ implies that $J_{x}$ is constant across the shock. Since there is no current in front of the shock, we conclude that $J_{x}=0$. Therefore the Joule heating term $J_{x} E_{x}$ in Eq. $(3 e)$ vanishes, and we can derive the electric field $E_{x}$ from Eq. $(4 a)$ :

$$
E_{x}=\frac{-\sigma_{H}\left(u \sin \chi_{b}-v \cos \chi_{b}\right)+\sigma_{P} w}{\sigma_{H} \cos ^{2} \chi_{b}+\sigma_{P} \sin ^{2} \chi_{b}} B \sin \chi_{b} .
$$

Moreover, the ordinary electrical conductivity is assumed to be zero in front of the shock $\left(\sigma_{e 1}=0\right)$ since we consider that there is no plasma upstream, and the ambient conductivity is negligible [3].

\subsection{Integration}

Substituting $E_{x}$ with Eq. (9) in the differential system (3a-e), and integrating with a discontinuity of $\gamma, \sigma_{e}, \sigma_{P}, \sigma_{H}$ therefore yields:

$$
\left\{\begin{array}{l}
{[\rho u]=0} \\
{[m u+p]=\left[\frac{4}{3} \mu \frac{\mathrm{d} u}{\mathrm{~d} x}\right]-\left(\sin \chi_{b} \int_{x_{1}}^{x_{2}} N \sigma_{P} u \mathrm{~d} x-\cos \chi_{b} \int_{x_{1}}^{x_{2}} N \sigma_{P} v \mathrm{~d} x+\cos ^{2} \chi_{b} \int_{x_{1}}^{x_{2}} K \sigma_{H} w \mathrm{~d} x\right) B^{2} \sin \chi_{b}} \\
m[v]=\left[\frac{4}{3} \mu \frac{\mathrm{d} v}{\mathrm{~d} x}\right]+\left(\sin \chi_{b} \int_{x_{1}}^{x_{2}} N \sigma_{P} u \mathrm{~d} x-\cos \chi_{b} \int_{x_{1}}^{x_{2}} N \sigma_{P} v \mathrm{~d} x+\cos ^{2} \chi_{b} \int_{x_{1}}^{x_{2}} K \sigma_{H} w \mathrm{~d} x\right) B^{2} \cos \chi_{b} \\
m[w]=\left[\frac{4}{3} \mu \frac{\mathrm{d} w}{\mathrm{~d} x}\right]+\left(\sin \chi_{b} \int_{x_{1}}^{x_{2}} K \sigma_{H} u \mathrm{~d} x-\int_{x_{1}}^{x_{2}} K \sigma_{P} w \mathrm{~d} x\right) B^{2} \cos ^{2} \chi_{b} \\
m\left[h+\frac{V^{2}}{2}\right]=\left[\frac{4}{3} \mu u \frac{\mathrm{d} u}{\mathrm{~d} x}+\frac{4}{3} \mu v \frac{\mathrm{d} v}{\mathrm{~d} x}+\frac{4}{3} \mu w \frac{\mathrm{d} w}{\mathrm{~d} x}\right]
\end{array}\right.
$$

with the constant normal mass flow rate:

$m=\rho u=\rho_{1} u_{1}=\rho_{2} u_{2}$,

and the weights $K$ and $N$ :

$$
\left\{\begin{array}{l}
K=\frac{\sigma_{e} \cos ^{2} \chi_{b}}{\sigma_{e} \cos ^{2} \chi_{b}+\sigma_{P} \sin ^{2} \chi_{b}} \\
N=\frac{\sigma_{e} \cos ^{2} \chi_{b}+\sigma_{C} \sin ^{2} \chi_{b}}{\sigma_{e} \cos ^{2} \chi_{b}+\sigma_{P} \sin ^{2} \chi_{b}}
\end{array}\right.
$$

The electrical conductivity $\sigma_{C}$, appearing in the expression of $N$, Eq. (12b), is nothing else that the electrical conductivity introduced by Cowling in geophysical problems [11], such that:

$\sigma_{C}=\sigma_{P}+\frac{\sigma_{H}^{2}}{\sigma_{P}}$

Like in our reference work without Hall effect [3], we shall make use of the following relations, resulting from mass conservation, (Eq. $2 a$ ), with the mass density characteristic scale $\mathscr{L}_{\rho}$, assumed constant across the shock:

$u=-\mathscr{L}_{\rho} \frac{\mathrm{d} u}{\mathrm{~d} x}, \quad \frac{1}{\mathscr{L}_{\rho}}=\frac{1}{\rho} \frac{\mathrm{d} \rho}{\mathrm{d} x}$, 
We are also led to define similar quantities $\mathscr{L}_{v}$ and $\mathscr{L}_{w}$, in order to express in the same way the derivatives of the velocity components $v$ and $w$, namely:

$v=\mathscr{L}_{v} \frac{\mathrm{d} v}{\mathrm{~d} x}, \quad w=\mathscr{L}_{v} \frac{\mathrm{d} w}{\mathrm{~d} x}$.

In contrast to the minus sign of Eq. (14), the plus sign in Eqs. (15a,b) is justified by the fact that the magnitudes of $v$ and $w$ increase through the shock, while that of $u$ decreases. As in the situation without Hall effect, the ordinary conductivity is assumed to satisfy a relation similar to (15), with the length scale $\mathscr{L}_{\sigma}$ :

$\sigma_{e}=\mathscr{L}_{\sigma} \frac{\mathrm{d} \sigma_{e}}{\mathrm{~d} x}$.

Following a thermodynamic argumentation, it is possible to relate $\mathscr{L}_{\sigma}$ and $\mathscr{L}_{\rho}$ (Sect. 2.4). Likewise, the conductivities $\sigma_{P}$ and $\sigma_{H}$ are also supposed to satisfy the relations:

$\sigma_{P}=\mathscr{L}_{\sigma} \frac{\mathrm{d} \sigma_{P}}{\mathrm{~d} x}, \quad \sigma_{H}=\mathscr{L}_{\sigma} \frac{\mathrm{d} \sigma_{H}}{\mathrm{~d} x}$.

By using the definitions of Eq. (17), the following calculation

$\frac{\mathrm{d} \sigma_{C}}{\mathrm{~d} x}=\left(1-\frac{\sigma_{H}^{2}}{\sigma_{P}^{2}}\right) \frac{\mathrm{d} \sigma_{P}}{\mathrm{~d} x}+\frac{2 \sigma_{H}}{\sigma_{P}} \frac{\mathrm{d} \sigma_{H}}{\mathrm{~d} x}=\frac{\sigma_{P}}{\mathscr{L}_{\sigma}}\left(1+\frac{\sigma_{H}^{2}}{\sigma_{P}^{2}}\right)=\frac{\sigma_{C}}{\mathscr{L}_{\sigma}}$

shows that Cowling's conductivity satisfies the same relation as $\sigma_{e}, \sigma_{p}$ and $\sigma_{H}$ through the shock, namely:

$\sigma_{C}=\mathscr{L}_{\sigma} \frac{\mathrm{d} \sigma_{C}}{\mathrm{~d} x}$.

These properties mean that the corresponding quantities are exponential functions of $x$. Moreover, the calculations detailed in Appendices 1 and 2 show that the integrals appearing in system (10) can be written:

$$
\left\{\begin{array}{l}
\int_{x_{1}}^{x_{2}} N \sigma_{P} u \mathrm{~d} x=\mathscr{L}_{\rho \sigma}\left[N \sigma_{P} u\right] \\
\int_{x_{1}}^{x_{2}} N \sigma_{P} v \mathrm{~d} x=\mathscr{L}_{v \sigma}\left[N \sigma_{P} v\right] \\
\int_{x_{1}}^{x_{2}} K \sigma_{H} w \mathrm{~d} x=\mathscr{L}_{w \sigma}\left[K \sigma_{H} w\right] \\
\int_{x_{1}}^{x_{2}} K \sigma_{H} u \mathrm{~d} x=\mathscr{L}_{\rho \sigma}\left[K \sigma_{H} u\right] \\
\int_{x_{1}}^{x_{2}} K \sigma_{P} w \mathrm{~d} x=\mathscr{L}_{w \sigma}\left[K \sigma_{P} w\right]
\end{array}\right.
$$

where, like in the situation without Hall effect, we have defined the compound length scales $\mathscr{L}_{\rho \sigma}, \mathscr{L}_{v \sigma}$ and $\mathscr{L}_{w \sigma}$ by the relations (87) in Appendix 2. Using all these results and notations, the integration of system (10) results in the following set of equations for the jumps:

$$
\left\{\begin{array}{l}
{[\rho u]=0} \\
{[m u+p]=\left[\frac{4}{3} \mu \frac{\mathrm{d} u}{\mathrm{~d} x}\right]-\left(\mathscr{L}_{\rho \sigma} \sin \chi_{b}\left[N \sigma_{P} u\right]-\mathscr{L}_{v \sigma} \cos \chi_{b}\left[N \sigma_{P}\right]+\mathscr{L}_{w \sigma} \cos ^{2} \chi_{b}\left[K \sigma_{H} w\right]\right) B^{2} \sin \chi_{b}} \\
m[v]=\left[\frac{4}{3} \mu \frac{\mathrm{d} v}{\mathrm{~d} x}\right]+\left(\mathscr{L}_{\rho \sigma} \sin \chi_{b}\left[N \sigma_{P} u\right]-\mathscr{L}_{v \sigma} \cos \chi_{b}\left[N \sigma_{P} v\right]+\mathscr{L}_{w \sigma} \cos ^{2} \chi_{b}\left[K \sigma_{H} w\right]\right) B^{2} \cos \chi_{b} \\
m[w]=\left[\frac{4}{3} \mu \frac{\mathrm{d} w}{\mathrm{~d} x}\right]+\left(\mathscr{L}_{\rho \sigma} \sin \chi_{b}\left[K \sigma_{H} u\right]-\mathscr{L}_{w \sigma}\left[K \sigma_{P} w\right]\right) B^{2} \cos ^{2} \chi_{b} \\
m\left[\frac{\gamma}{\gamma-1} \frac{p}{\rho}+\frac{u^{2}+v^{2}+w^{2}}{2}\right]=\left[\frac{4}{3} \mu u \frac{\mathrm{d} u}{\mathrm{~d} x}+\frac{4}{3} \mu \nu \frac{\mathrm{d} v}{\mathrm{~d} x}+\frac{4}{3} \mu w \frac{\mathrm{d} w}{\mathrm{~d} x}\right]
\end{array}\right.
$$


The usual boundary conditions used for the derivation of the Rankine-Hugoniot relations assume that the $x$ derivatives of $u$ and $v$ (and here $w$ ) vanish at infinity, before and behind the shock, and therefore we also neglect the viscous terms involving $\mathrm{d} u / \mathrm{d} x, \mathrm{~d} v / \mathrm{d} x$ and $\mathrm{d} w / \mathrm{d} x$. Nevertheless, these conditions do not strictly hold when the shock is curved [3]. This assumption implies in particular that the total energy is unchanged across the shock (Eq. (21e)). Moreover, Eq. (21c) shows that the transverse velocity $v$ has a jump if the magnetic field does not lie in the shock plane $\left(\chi_{b} \neq \pm 90^{\circ}\right)$, and Eq. $(21 d)$ shows that there is a third component $w$ due the Hall effect, causing a swirl in cylindrical geometry [25].

\subsection{Algebraic system}

Once the viscous terms are removed, the system (21) can be simply written as:

$$
\left\{\begin{array}{l}
{[\rho u]=0} \\
{[m u+p]=-\left(\mathscr{L}_{\rho \sigma} \sin \chi_{b}\left[N \sigma_{P} u\right]-\mathscr{L}_{v \sigma} \cos \chi_{b}\left[N \sigma_{P} v\right]+\mathscr{L}_{w \sigma} \cos ^{2} \chi_{b}\left[K \sigma_{H} w\right]\right) B^{2} \sin \chi_{b}} \\
m[v]=\left(\mathscr{L}_{\rho \sigma} \sin \chi_{b}\left[N \sigma_{P} u\right]-\mathscr{L}_{v \sigma} \cos \chi_{b}\left[N \sigma_{P} v\right]+\mathscr{L}_{w \sigma} \cos ^{2} \chi_{b}\left[K \sigma_{H} w\right]\right) B^{2} \cos \chi_{b} \\
m[w]=\left(\mathscr{L}_{\rho \sigma} \sin \chi_{b}\left[K \sigma_{H} u\right]-\mathscr{L}_{w \sigma}\left[K \sigma_{P} w\right]\right) B^{2} \cos ^{2} \chi_{b} \\
{\left[\frac{\gamma}{\gamma-1} \frac{p}{\rho}+\frac{u^{2}+v^{2}+w^{2}}{2}\right]=0}
\end{array}\right.
$$

This is a nonlinear algebraic system for the five unknown jumps $[p],[\rho],[u],[v],[w]$, or, equivalently, the five unknown downstream values $p_{2}, \rho_{2}, u_{2}, v_{2}, w_{2}$, since the upstream values are known.

By developing the brackets in Eqs. (22), rearranging terms and eliminating suitable quantities, we can show that the downstream velocity components can be written as:

$$
\left\{\begin{array}{l}
u_{2}=a\left(u_{1}+\frac{p_{1}-p_{2}}{m}\right)+b v_{1} \\
v_{2}=c\left(u_{1}+\frac{p_{1}-p_{2}}{m}\right)+d v_{1} \\
w_{2}=f u_{2}
\end{array}\right.
$$

A summary of the demonstration is given in Appendix 3, and it is shown therein how we are led to define three microscopic interactions parameters, or Stuart numbers, $S_{\rho \sigma}, S_{v \sigma}, S_{w \sigma}$, from the three length scales $\mathscr{L}_{\rho \sigma}, \mathscr{L}_{v \sigma}$, $\mathscr{L}_{w \sigma}$, Eqs. (89). Raw expressions for the five fundamental coefficients $a, b, c, d$, and $f$ are obtained after some tedious algebra. By neglecting small quantities proportional to $S_{\rho \sigma} S_{w \sigma}$, they can be finally simplified as:

$$
\left\{\begin{array}{l}
a \approx \frac{1+N_{2} \tilde{\sigma}_{P 2} S_{v \sigma} \cos ^{2} \chi_{b}}{1+N_{2} \tilde{\sigma}_{P 2}\left(S_{\rho \sigma} \sin ^{2} \chi_{b}+S_{v \sigma} \cos ^{2} \chi_{b}\right)} \\
b \approx \frac{N_{2} \tilde{\sigma}_{P 2} S_{v \sigma} \sin \chi_{b} \cos \chi_{b}}{1+N_{2} \tilde{\sigma}_{P 2}\left(S_{\rho \sigma} \sin ^{2} \chi_{b}+S_{v \sigma} \cos ^{2} \chi_{b}\right)} \\
c \approx \frac{N_{2} \tilde{\sigma}_{P 2} S_{\rho \sigma} \sin \chi_{b} \cos \chi_{b}}{1+N_{2} \tilde{\sigma}_{P 2}\left(S_{\rho \sigma} \sin ^{2} \chi_{b}+S_{v \sigma} \cos ^{2} \chi_{b}\right)} \\
d \approx \frac{1+N_{2} \tilde{\sigma}_{P 2} S_{\rho \sigma} \sin ^{2} \chi_{b}}{1+N_{2} \tilde{\sigma}_{P 2}\left(S_{\rho \sigma} \sin ^{2} \chi_{b}+S_{v \sigma} \cos ^{2} \chi_{b}\right)}
\end{array}\right.
$$

and:

$f=\frac{K_{2} \tilde{\sigma}_{H 2} S_{\rho \sigma} \sin \chi_{b} \cos ^{2} \chi_{b}}{1+K_{2} \tilde{\sigma}_{P 2} S_{w \sigma} \cos ^{2} \chi_{b}}$ 
where the tilde $\sim$ means that the conductivities $\sigma_{P}, \sigma_{H}$ and $\sigma_{C}$ are normalized to $\sigma_{e}$ :

$\tilde{\sigma}_{P 2}=\frac{\sigma_{P 2}}{\sigma_{e 2}}, \quad \tilde{\sigma}_{H 2}=\frac{\sigma_{H 2}}{\sigma_{e 2}}, \quad \tilde{\sigma}_{C 2}=\frac{\sigma_{C 2}}{\sigma_{e 2}}$.

The expressions of the four coefficients $a, b, c, d$, Eqs. (24), are formally identical with those obtained without Hall effect [3], except for the presence of the weights $K_{2}, N_{2}$, and of normalized conductivities. Like in [3], the coefficients $a$ and $d$ are of order unity, while $b$ and $c$ are quite small in magnitude (Table 4).

By elimination of $p$ and $\rho$, we can recast velocity jumps $(23 a, b, c)$ into the simple form:

$\left\{\begin{array}{l}u_{2}=\varepsilon u_{1} \\ v_{2}=\eta v_{1} \\ w_{2}=f \varepsilon u_{1}\end{array}\right.$

where we have used, like in our previous work [3], the magnetic parameter $\eta$ :

$\eta=d-\frac{b c}{a}+\frac{c}{a} \varepsilon \cot \chi_{1}$

in addition to the classical ratio $\varepsilon$ of mass densities:

$\varepsilon=\frac{\rho_{1}}{\rho_{2}}$.

It is interesting to notice the symmetry of relations $(27 a, b)$, with $\varepsilon$ and $\eta$ playing similar role. The transverse velocity $v$ is theoretically discontinuous in the presence of a magnetic field $(\eta \neq 1)$, and the third velocity component $w$ appears behind the shock in the presence of Hall effect $(f \neq 0)$. Without Hall effect $\left(\beta_{e}=0, s=0\right.$, $\left.\sigma_{P}=\sigma_{e}, \sigma_{H}=0, f=0\right)$, Eq. (27c) shows that $w_{2}=0$, and without magnetic field $(B=0, a=d=\eta=1, b=c=f$ $=0$ ), we obtain $v_{2}=v_{1}$ from Eq. (27b), i.e. continuity of $v$.

\subsection{Length scales}

This short section is devoted to the choice of the different length scales that are a fundamental input of our model. First, the relationship between $\mathscr{L}_{\rho}$ and $\mathscr{L}_{\sigma}$, established on the basis of thermodynamic arguments in [3], is supposed to be still valid:

$\frac{\mathscr{L}_{\rho}}{\mathscr{L}_{\sigma}} \approx \alpha(\gamma-1-1 / \zeta)$

The parameter $\zeta$, being defined as the ratio:

$\zeta=\frac{\mathscr{L}_{16}}{\mathscr{L}_{\rho}}$

where $\mathscr{L}_{16}$ denotes the length scale of molar mass, takes into account the deviation from equilibrium within the shock. As shown in [3] from estimations of the Damköhler number $N_{D}$ [8], chemical non-equilibrium is probably more developed at $65 \mathrm{~km}\left(N_{D}<1\right)$ than at $40 \mathrm{~km}\left(N_{D}>1\right)$, so that we are led to choose $\zeta \approx 10$, and, with $\gamma \approx 1.15$ we obtain: $\mathscr{L}_{\sigma} \approx 10 \mathscr{L}_{\rho}$. More generally, the problem of thermochemical non-equilibrium will be examined in Sect. 3.4.3. A simple estimate of $\mathscr{L}_{\rho}$ is given by the following expression [3]:

$\mathscr{L}_{\rho}=\frac{v_{1}}{c_{s 1}}$.

Numerical estimates of the upstream kinematic viscosity $v_{1}$ and sound speed $c_{s 1}$ are displayed in Table 2.

Following our previous hypotheses concerning the length scales, we shall suppose that $\mathscr{L}_{v}=\mathscr{L}_{\rho}$ [3]. In addition, the transverse components $v$ and $w$ playing similar roles, we shall assume that $\mathscr{L}_{w}=\mathscr{L}_{v}$. This enables one to estimate the compound length scales defined by Eqs. (87):

$\mathscr{L}_{\rho \sigma} \approx-1.1 \mathscr{L}_{\rho}, \quad \mathscr{L}_{v \sigma} \approx \mathscr{L}_{w \sigma} \approx 0.91 \mathscr{L}_{\rho}$.

We notice that $\mathscr{L}_{\rho \sigma}$ can be negative $\left(\mathscr{L}_{\rho}>0\right)$. 


\subsection{General solutions}

Like in our previous work [3], the jumps of the pressure and mass density ratio are eventually found as solutions of quadratic equations, as shown in Appendix 3. Thus, on one hand, by eliminating $\rho, u, v, w$, we obtain the equation for the pressure ratio:

$A_{2} X^{2}+2 A_{1} X+A_{0}=0$,

with the unknown:

$X=\frac{p_{2}}{p_{1}}-1$,

and the coefficients:

$\left\{\begin{array}{l}A_{2}=\frac{1}{\gamma_{1} M_{1}^{2} \cos ^{2} \chi_{1}}\left\{\left(1+f^{2}\right) a^{2}+c^{2}-2 \frac{\gamma_{2}}{\gamma_{2}-1} a\right\} \\ A_{1}=\frac{\gamma_{2}}{\gamma_{2}-1}\left(a+b \tan \chi_{1}-\frac{a}{\gamma_{1} M_{1}^{2} \cos ^{2} \chi_{1}}\right)-\left\{\left(1+f^{2}\right) a^{2}+c^{2}\right\}-\left\{\left(1+f^{2}\right) a b+c d\right\} \tan \chi_{1} \\ A_{0}=\gamma_{1} M_{1}^{2}\left[\left\{\left(1+f^{2}\right)\left(a+b \tan \chi_{1}\right)^{2}+\left(c+d \tan \chi_{1}\right)^{2}\right\} \cos ^{2} \chi_{1}-1\right]+\frac{2 \gamma_{2}}{\gamma_{2}-1}\left(a+b \tan \chi_{1}\right)-\frac{2 \gamma_{1}}{\gamma_{1}-1}\end{array}\right.$

On the other hand, by eliminating $p, u, v, w$, we obtain the equation for the mass density ratio:

$C_{2} Y^{2}+2 C_{1} Y+C_{0}=0$,

with the unknown:

$Y=\frac{\rho_{2}}{\rho_{1}}$,

and the coefficients:

$\left\{\begin{array}{l}C_{2}=\frac{2 \gamma_{1}}{\gamma_{1}-1}-\left(d-\frac{b c}{a}\right)^{2} \gamma_{1} M_{1}^{2} \sin ^{2} \chi_{1}+\gamma_{1} M_{1}^{2} \\ C_{1}=-\left[\frac{\gamma_{2}}{\gamma_{2}-1}\left\{1+\left(1+\frac{b}{a} \tan \chi_{1}\right) \gamma_{1} M_{1}^{2} \cos ^{2} \chi_{1}\right\}+\left(d-\frac{b c}{a}\right) \frac{c}{a} \gamma_{1} M_{1}^{2} \sin \chi_{1} \cos \chi_{1}\right] \\ C_{0}=\left(\frac{2 \gamma_{2}}{\gamma_{2}-1} \frac{1}{a}-1-\frac{c^{2}}{a^{2}}-f^{2}\right) \gamma_{1} M_{1}^{2} \cos ^{2} \chi_{1}\end{array}\right.$

In consistency with our reference work without Hall effect, for the pressure ratio, we chose the solution:

$X=\frac{-A_{1}-\sqrt{A_{1}^{2}-A_{0} A_{2}}}{A_{2}}$,

while for the mass density ratio, we chose the solution:

$Y=\frac{-C_{1}+\sqrt{C_{1}^{2}-C_{0} C_{2}}}{C_{2}}$,

provided the discriminants under the radical signs are positive.

Besides, we can check that the two unknowns $X$ and $Y$ are not independent, and that the relation [3]:

$X=\frac{\gamma_{1} M_{1}^{2} \cos ^{2} \chi_{1}}{a}\left(a+b \tan \chi_{1}-\frac{1}{Y}\right)$

derived from Eq. (92a), is still formally valid, as well as the velocity ratio:

$\frac{V_{2}^{\prime}}{V_{1}}=\sqrt{\eta^{2} \sin ^{2} \chi_{1}+\varepsilon^{2} \cos ^{2} \chi_{1}}$.

From Eq. $(27 a, b)$, can we easily derive that the velocity angle $\chi_{2}$ behind the shock is such that:

$\tan \chi_{2}=\frac{\eta}{\varepsilon} \tan \chi_{1}$. 
It is remarkable that this relation, describing the deflection of streamlines by the shock, is analogous to the sinelaw of refraction in optics, the ratio $\eta / \varepsilon$ playing here the role of the refraction index [3].

The five coefficients $a, b, c, d, f$ are known once the primary parameters $\left(V_{1}, B, \gamma_{1}, \chi_{1}, \chi_{b}\right)$ are specified and the two quantities $\left(\mathscr{L}_{\rho}, m\right)$ are calculated from the physical conditions upstream. In contrast, the six coefficients $A_{0}, A_{1}, A_{2}, C_{0}, C_{1}, C_{2}$ of the quadratic equations (34) and (37) depend implicitly on $\gamma_{2}$ and $\sigma_{e 2}$, so that the solutions $X$ and $Y$ must be searched through an iterative procedure, the so-to-say shock fitting, developed in the Sect. 3 below.

\subsection{Particular cases}

\subsubsection{Non-magnetic case}

Without magnetic field $(B=0, a=d=1, b=c=f=0)$ and without jump of $\gamma\left(\gamma_{1}=\gamma_{2}=\gamma\right)$, we recover the classical Rankine-Hugoniot explicit solutions, Eqs. (1), valid for a perfect gas or an inert gas (such as argon, neon, krypton, xenon). Otherwise $\left(\gamma_{1} \neq \gamma_{2}\right)$, we obtain aerodynamic jumps due to molecular dissociations, still solutions of the quadratic equations (34) and (37).

These cases being extensively described in our previous work [3], we shall not deal in detail with them here. Nevertheless, we recall that, using the same methodology as ours and including the effects of viscosity on a hydrodynamic $(B=0)$ normal shock $\left(\chi_{1}=0\right)$ for a perfect gas $\left(\gamma_{1}=\gamma_{2}\right)$, a recent work leads to a quadratic equation similar to our Eq. (37), with additional terms in the coefficients $C_{0}, C_{1}, C_{2}$, equal to the reciprocals of upstream and downstream kinetic Reynolds numbers $R e_{1}$ and $R e_{2}$ [10]. In the limit of non-viscous flow $\left(R e_{1} \rightarrow+\infty\right.$ and $\left.R e_{2} \rightarrow+\infty\right)$, this author's equation and our Eq. (37) with the coefficients (39), simplified accordingly, become identical.

\subsubsection{Magnetic case without Hall effect}

In the absence of Hall effect $\left(B \neq 0, \beta_{e 2}=0, s_{2}=0, \sigma_{P 2}=\sigma_{e 2}, \sigma_{H 2}=0, \sigma_{C 2}=\sigma_{e 2}\right)$, the coefficients $K$ and $N$, defined by Eqs. (12), have the following values behind the shock:

$$
K_{2}=\cos ^{2} \chi_{b}, \quad N_{2}=1,
$$

and the coefficients $a, b, c, d, f$, defined by the relations (24) and (25), become:

$$
\left\{\begin{array}{l}
a \approx \frac{1+S_{v \sigma} \cos ^{2} \chi_{b}}{1+S_{\rho \sigma} \sin ^{2} \chi_{b}+S_{v \sigma} \cos ^{2} \chi_{b}} \\
b \approx \frac{S_{v \sigma} \sin \chi_{b} \cos \chi_{b}}{1+S_{\rho \sigma} \sin ^{2} \chi_{b}+S_{v \sigma} \cos ^{2} \chi_{b}} \\
c \approx \frac{S_{\rho \sigma} \sin \chi_{b} \cos \chi_{b}}{1+S_{\rho \sigma} \sin ^{2} \chi_{b}+S_{v \sigma} \cos ^{2} \chi_{b}} \\
d \approx \frac{1+S_{\rho \sigma} \sin ^{2} \chi_{b}}{1+S_{\rho \sigma} \sin ^{2} \chi_{b}+S_{v \sigma} \cos ^{2} \chi_{b}} \\
f=0
\end{array}\right.
$$

which are the expressions we derived without Hall effect [3]. Therefore, we shall also obtain the same coefficients for the quadratic Eqs. (34) and (37), and finally recover the solutions $X$ and $Y$ of paper [3].

Since in that case, we simply have: $w_{2}=0$ and $E_{x 2}=0$, we directly obtain from Eq. (4) $: J_{x 2}=0, J_{y 2}=0$, so that the current density is reduced to its Pedersen component $J_{z 2}$, such that:

$J_{z 2}=\sigma_{e 2}\left(u_{2} \sin \chi_{b}-v_{2} \cos \chi_{b}\right) B$

and using Eqs. $(27 a, b)$ and (97) :

$J_{z 2}=\sigma_{e 2} V_{1} B\left(\varepsilon \cos \chi_{1} \sin \chi_{b}-\eta \sin \chi_{1} \cos \chi_{b}\right)$. 


\subsubsection{Quasi-normal shock}

This case $\left(\chi_{1}= \pm 90^{\circ}\right)$, formally equivalent to $M_{1} \rightarrow 0$, yields for the pressure ratio:

$X\left\{\left(\left(1+f^{2}\right) a^{2}+c^{2}-2 a \frac{\gamma_{2}}{\gamma_{2}-1}\right) X-2 a \frac{\gamma_{2}}{\gamma_{2}-1}\right\}=0$,

and for the mass density ratio:

$Y\left(\left[\frac{2 \gamma_{1}}{\gamma_{1}-1}+\left\{1-\left(d-\frac{b c}{a}\right)^{2}\right\} \gamma_{1} M_{1}^{2}\right] Y-2 \frac{\gamma_{2}}{\gamma_{2}-1}\right)=0$

Rejecting the trivial solutions $(X=0$ and $Y=0)$, we obtain the new solutions:

$X=\frac{2 \frac{\gamma_{2}}{\gamma_{2}-1} a}{\left(1+f^{2}\right) a^{2}+c^{2}-2 \frac{\gamma_{2}}{\gamma_{2}-1} a}$,

and

$Y=\frac{\frac{2 \gamma_{2}}{\gamma_{2}-1}}{\frac{2 \gamma_{1}}{\gamma_{1}-1}+\left\{1-\left(d-\frac{b c}{a}\right)^{2}\right\} \gamma_{1} M_{1}^{2}}$.

The pressure ratio is therefore written as:

$X+1=\frac{\left(1+f^{2}\right) a^{2}+c^{2}}{\left(1+f^{2}\right) a^{2}+c^{2}-2 \frac{\gamma_{2}}{\gamma_{2}-1} a}$.

With typical values at 40 and $65 \mathrm{~km}$ (Table 4), which imply $a \approx 1, c \ll<1$ and $f \ll<$, we can further simplify this latter expression into:

$X+1 \approx-\frac{\gamma_{2}-1}{\gamma_{2}+1}$.

Therefore $X<0$, so that case is unphysical, like in aerodynamics, since the shock is supersonic $\left(M_{1}>1\right)$.

\subsubsection{Normal magnetic field}

For a normal magnetic field $\left(\chi_{b}=0\right.$ or $\left.180^{\circ}\right)$, the coefficients $K$ and $N$ behind the shock take the values:

$K_{2}=1, \quad N_{2}=1$,

and the coefficients $a, b, c, d, f$ become:

$a=1, \quad b=0, \quad c=0, \quad d \approx \frac{1}{1+\tilde{\sigma}_{P 2} S_{v \sigma}}, \quad f=0$.

This implies for (42):

$X=\gamma_{1} M_{1}^{2} \cos ^{2} \chi_{1}\left(1-\frac{1}{Y}\right)$

and for (28):

$\eta=d$.

Therefore, we recover the same pressure-density relationship as in aerodynamics, but there is a jump of tangential velocity $v$. Moreover, these results are formally identical with the case without Hall effect, except for the factor $\tilde{\sigma}_{P 2}$ appearing in the constant $d$. 


\subsubsection{Tangential magnetic field}

On the other hand, for a tangential magnetic field $\left(\chi_{b}= \pm 90^{\circ}\right)$, the coefficients $K$ and $N$ behind the shock have the values:

$$
K_{2}=0, \quad N_{2}=\frac{\sigma_{C 2}}{\sigma_{P 2}},
$$

and the coefficients $a, b, c, d$, $f$ become:

$a \approx \frac{1}{1+\tilde{\sigma}_{C 2} S_{\rho \sigma}}, \quad b=0, \quad c=0, \quad d=1, \quad f=0$.

This implies for (42):

$X=\gamma_{1} M_{1}^{2} \cos ^{2} \chi_{1}\left(1-\frac{1}{a Y}\right)$,

and for (28):

$\eta=1$.

Therefore, the tangential velocity $v$ (Eq. $(27 b)$ ) is continuous through the shock, like in aerodynamics, but the pressure-density relationship (61) involves an MHD correcting factor. Moreover, these results are formally identical with the case without Hall, except for the factor $\tilde{\sigma}_{C 2}$ appearing in the constant $a$, and an induced electric field $E_{x 2}$, Eq. (9), is produced, that can be written here:

$E_{x 2}=-\frac{\sigma_{H 2}}{\sigma_{P 2}} u_{2} B$

In conclusion, these two latter cases are hybrid, since they combine aerodynamic and MHD features. This situation is similar to that already encountered without Hall effect [3].

\section{Shock fitting}

\subsection{Method}

The present situation of a plane oblique shock is relatively simple, since only the isentropic exponent $\gamma_{2}$ and the ordinary conductivity $\sigma_{e 2}$ behind the shock need to be iterated (Fig. 2). The twofold convergence test at step $n$ is therefore taken as:

$\left|\frac{\gamma_{2}^{(n)}-\gamma_{2}^{(n-1)}}{\gamma_{2}^{(n-1)}}\right|<\varpi, \quad\left|\frac{\sigma_{e 2}^{(n)}-\sigma_{e 2}^{(n-1)}}{\sigma_{e 2}^{(n-1)}}\right|<\varpi$,

$(64 a, b)$

with the threshold value, $\varpi=10^{-8}$. Initial values are chosen such that $\gamma_{2}^{(0)}=1.4, \sigma_{e 2}(0)=100 \mathrm{~S} \mathrm{~m}^{-1}$. The convergence depends on the angles of velocity $\chi_{1}$ and magnetic field $\chi_{b}$, and it is attained typically in 20 to 40 iterations.

For the sake of simplicity in the present analytic model, we consider, like in our previous paper, that the air plasma is in thermal equilibrium, and that chemical non-equilibrium is implicitly modeled by means of the parameter $\zeta$ [3]. Therefore, we use the same thermodynamic model, based on correlations, that provides the plasma temperature $T$ via a thermal state equation, $T(p, \rho)$, and the isentropic exponent $\gamma$ via a caloric state equation, $\gamma(p, \rho)$, both as functions of the plasma pressure $p$ and mass density $\rho$ [3][45]. More recent correlations in air plasma in equilibrium have been published and they could be used for future work [13][14]. The validity of equilibrium assumptions will be discussed below, in Sect. 3.4.

When the Hall effect is developed in the MHD interaction, the primary parameter cannot be the Stuart number $S_{\rho}$ previously used [3] - since the Hall parameters cannot be cast as functions of $S_{\rho}$ alone -, but instead, the magnetic field strength $B$ itself. If we want to keep on calculating the ordinary electrical conductivity $\sigma_{e}$ by means of the power law, Eq. (8), in order to be consistent with our previous work, we are led to first extract the electronic density by iterating Spitzer's relation, Eq. $(5 a)$, knowing $\sigma_{e 2}$, and then estimate the Hall parameters $\beta_{e 2}, \beta_{i 2}$ and slip factor $s_{2}$ (Eqs. (6) and (7)) that are necessary for the calculation of the Pedersen and Hall conductivities, Eq. $(5 b, c)$. The resulting algorithm is depicted in Fig. 3. 


\subsection{Results}

We illustrate the behavior of the shock front with the calculation of physical quantities behind the shock for two specific geometrical configurations, namely case $1\left(\chi_{1}=20^{\circ} ; \chi_{b}=170^{\circ}\right)$ and case $2\left(\chi_{1}=60^{\circ} ; \chi_{b}=150^{\circ}\right)$, using physical ambient conditions at the altitudes of 40 and $65 \mathrm{~km}$ (Table 2), without and with Hall effect. The isentropic exponent in the ambient air ahead of the shock is $\gamma_{1}=1.4$.

Convergence plots of the isentropic exponent $\gamma_{2}$ and ordinary conductivity $\sigma_{e 2}$ are displayed (Fig. 4) for case 2, with physical conditions at $65 \mathrm{~km}$ height, without (Fig. $4 a, b$ ) and with (Fig. $4 c, d$ ) Hall effect. They show that limits are reached after approximately 11 iterations and 25 iterations respectively. Moreover, it can be noticed that the convergence profile is more oscillatory with Hall effect $(c, d)$ than without $(a, b)$, since that situation is more complex and needs more iterations.

The results at $40 \mathrm{~km}$ are not plotted here because, the MHD interaction within the shock being quite small, the impact of the Hall effect is hardly noticeable, as it can be checked by comparing the data of Table 3, without Hall effect, and Table 4, with Hall effect. Likewise, case 2 is preferred to case 1 because it shows better the efficiency of the Hall effect, and expectedly, it corresponds approximately to the position along the OREX reentry vehicle where the Hall generator is positioned [20][21].

In contrast, at an altitude of $65 \mathrm{~km}$, for the geometrical case 2, the variations of the pressure (Fig. 5), mass density (Fig. 6) and temperature (Fig. 7) ratios, isentropic exponent (Fig. 8) and ordinary electrical conductivity (Fig. 9) as functions of $M_{1}$ and $B$ show the following striking features.

In the absence of Hall effect (or when the Hall effect is neglected), the pressure (Fig. $5 a$ ), the mass density (Fig. 6a) and the isentropic exponent (Fig. 8a) are reduced by the MHD interaction as $B$ increases, while the temperature (Fig. 7a) and the electrical conductivity (Fig. 9a) are raised. As can be clearly seen on the mass density ratio (Fig. $6 a$ ), the abscissa of the hump decreases as $B$ increases, from $M_{1} \approx 25$ for $B=0 \mathrm{~T}$ to $M_{1} \approx 19$ for $B=1 \mathrm{~T}$. The origin of this hump, due to a resonance of molecular dissociations, is explained in detail in [3].

In the presence of Hall effect (Figs $5 b, 6 b, 7 b, 8 b$ and $9 b$ ), the MHD interaction is nearly cancelled: all the curves corresponding to the various values of the magnetic field $B$, below the Mach number $M_{1} \approx 22$, are coincident with the aerodynamic curve $(B=0)$. This degeneracy is seen on most of the variables describing the system (including velocity components $u_{2}$ and $v_{2}$, not displayed here), except the Pedersen current $J_{z 2}$, since we find that $E_{x 2} \rightarrow 0$ from (9) and $J_{z 2} \rightarrow 0$ from (4c) as $B \rightarrow 0$.

Actually, the Pedersen current $J_{z 2}$ (Fig. 10) exhibits an intermediary behavior. In the absence of Hall effect, it reaches values of a few hundred $\mathrm{kA} \mathrm{m}^{-2}$ at hypersonic speeds, while it is 1000 times smaller in the presence of Hall effect. The resulting MHD force $J_{22} B$ in the $(x, y)$ plane is therefore reduced in the same proportion.

The electric field $E_{x 2}$, Eq. (9) (Fig. 11a), essentially due to the Hall effect, is expected to produce small potential drops $U$ of a few millivolts $(U \approx 35.5 \times 0.359=12.7 \mathrm{mV}$ at Mach 23) perpendicular to the shock. The transverse velocity $w_{2}$, also induced by the Hall effect (Fig. 11b), amounts to a few $\mathrm{m} \mathrm{s}^{-1}$ and it is known that it may cause a swirling motion in an axisymmetric flow [25]. These quantities increase in magnitude with $B$ and $M_{1}$. Everything happens as if the Hall effect converted kinetic energy from the $(\boldsymbol{V}, \boldsymbol{B})$ plane to the perpendicular direction. Moreover, it can be noticed that the influence of the magnetic field on $J_{z}$ (Fig. 10b) and $w_{2}$ (Fig. 11b) becomes sensitive above $M_{1} \approx 10$.

It is also worth plotting the electronic Hall parameter $\beta_{e 2}$ and the slip factor $s_{2}$, that enable one to estimate the efficiency of the Hall effect, and to extrapolate its behavior as the magnetic field strength $B$ increases (Fig. 12). It could be noticed that the Hall parameters are decreasing functions of the upstream Mach number $M_{1}$, and that they are very large at small velocity, thus indicating that the Hall effect is more developed in supersonic regime.

At Mach 23, with $B=1 \mathrm{~T}$, we see on these plots that $\beta_{e 2} \approx 100$ and $s_{2} \approx 30$. Thus, it is noticeable that the slip factor is fairly large, though it is often neglected in numerical models [5][6][35][36]. Moreover, we shall see below how it leads to a limit value for the Laplace force and helps to explain the saturation of MHD interaction produced by the Hall effect as the magnetic field increases.

The present situation may be put in relation with the Hall effect acting in the blunt-body problem: the flow in the shock layer and the bow shock stand-off are substantially altered when the body wall is electrically insulating. On the opposite, when the body wall is electrically conducting, it is shown that the shock distance is equal to that of the aerodynamic flow without magnetic field for moderate magnetic fields [36].

\subsection{Discussion}

In the present section, our analytical model of resistive magnetohydrodynamic shock without Hall effect [3] has been extended to include a Hall effect. The same simplified approach, applied to a plane oblique shock neglects Ohmic and viscous dissipations, and pressure effects on the electrical conductivity. For consistency with our previous work, the conductivity has been calculated with a power law of temperature and the Hall parameters have been estimated by inversion of Spitzer's formula, Eq. ( $5 a$ ), yielding the electron density. 
The first results produced can be analyzed as follows. It clearly appears that the Hall effect develops counter to the MHD processes within the shock and tends to annihilate any magnetic action on pressure, mass density and temperature, resulting in the conditions of an aerodynamic, non-magnetic, flow for incident Mach numbers $M_{1} \leq 22$.

The variation of the total current density $J$ :

$J=\sqrt{J_{x}^{2}+J_{y}^{2}+J_{z}^{2}}$

as a function of the magnetic field strength $B$ in the presence of Hall effect (Fig. 13b) shows that $J$ has a sharp increase at the origin $(B=0)$ and then slowly decreases from a maximum to zero. Moreover, the smaller level of case 2, compared to that of case 1, indicates that the Hall effect is more efficient in case 2.

In the absence of Hall effect, $J$ is reduced to its Pedersen component $J_{z}$ since $J_{x}=J_{y}=0$, but it is much larger, and it would increase without limit since there is no Ohmic dissipation (Fig. 13a). The sharp increase of case 2 could not be drawn on the same plot, but it lies much above the profile of case 1, confirming that MHD processes are much more efficient in case 2. Furthermore, by comparing Table 3 and Table 4 at $65 \mathrm{~km}$, we see that the microscopic interaction parameter $S_{\rho}$ is lowered by the Hall effect.

So, we could simply conclude that "the more efficient the MHD action, the more efficient the Hall reaction". Such a process could be put in parallel with various feedback processes, such as Newton's third law in mechanics, Lenz's law in electromagnetism, and Le Chatelier's law in physical chemistry [31].

As already noticed above, this behavior in the shock front can be put in relation with that of the flow in the shock layer, between the shock and the body wall, in the blunt body problem [36]. Like in the present work, the intensity of electric currents with an insulating body wall may amount to several tens of $\mathrm{kA} \mathrm{m}^{-2}$ [6].

As a consequence, it can be shown that the Laplace force $F$ tends toward a limit as $B$ increases. Let us basically define the interaction parameter $S_{F}$ as the ratio of the force $F$ over the inertial force $F_{I}$, namely:

$S_{F}=\frac{F}{F_{I}}$.

The derivations of $F$ and $F_{I}$ are detailed in Appendix 4 and 5 respectively. As $B$ becomes indefinitely large, using Eqs (112) and (118) respectively, we obtain $S_{F}$ such that:

$S_{F} \underset{B \rightarrow+\infty}{\propto} \frac{\sigma_{e 2} B^{2} \mathscr{L}_{\rho}\left|\varepsilon \sin \chi_{b} \cos \chi_{1}-\eta \sin \chi_{1} \cos \chi_{b}\right|}{s_{2} \rho_{1} V_{1} \cos ^{2} \chi_{1}}$.

This latter relation can be cast into the form:

$S_{F} \underset{B \rightarrow+\infty}{\propto} S_{\rho} \frac{\left|\varepsilon \sin \chi_{b} \cos \chi_{1}-\eta \sin \chi_{1} \cos \chi_{b}\right|}{s_{2} \cos \chi_{1}}$,

where $s_{2}$ is the slip factor defined by Eq. (7), and $S_{\rho}$ denotes the usual Stuart number:

$S_{\rho}=\frac{\sigma_{e 2} B^{2} \mathscr{L}_{\rho}}{\rho_{1} V_{1} \cos \chi_{1}}$

As $B$ tends to infinity, we have: $S_{\rho} \sim B^{2}$ and $s_{2} \sim B^{2}$, so that, from (68), we conclude that $S_{F}$ tends toward a finite limit $S_{F \infty}$. It clearly depends on the geometrical case, as can be checked from the relation (67) and from the plots (Fig. 14b), whereas the usual interaction parameter $S_{\rho}$ increases indefinitely, and has approximately the same value for both cases (Fig. 14a). This is due to the fact that the electrical conductivity $\sigma_{e 2}$, closely related to the temperature $T_{2}$ by Eq. (8), varies approximately as $\cos \chi_{1}$, like in the absence of Hall effect [3].

Magnetic fields as large as a few tens of tesla would be necessary for the saturation effect to appear. Presently, such magnetic fields are actually produced for various laboratory experiments devoted to fundamental physics [1], and especially for the study of the quantum Hall effect in solids [41].

\subsection{Assessment}

\subsubsection{Interaction parameter}

The discussion above shows that using the common interaction parameter $S_{\rho}$ defined by Eq. (69) may be misleading and that the non-dimensional number $S_{F}$ based on the Laplace force, Eq. (66) is better suited. Another definition of the Stuart number $S_{H}$ based on $\beta_{e}$ has been proposed, but without ion slip factor, leading to $S_{H} \sim B$ at infinity [35]. Since this problem occurs locally, for a bow shock, we would be led to define an average 
interaction parameter, either as the integral of the "local" interaction parameter over the volume of MHD interaction [16]:

$\bar{S}_{Q}=\frac{1}{A_{b}} \int_{\vartheta \vartheta} \frac{\sigma_{e f f} B^{2}}{\rho_{1} V_{1}} \mathrm{~d} \vartheta$,

where $\sigma_{\text {eff }}$ is an effective electrical conductivity and $A_{b}$ the area of the frontal cross section, or as the integral of the "local" Laplace force over the same volume [5][18]:

$\bar{S}_{F}=\frac{1}{\rho_{1} V_{1}^{2} R_{b}^{2}} \int_{\vartheta}|\boldsymbol{F}| \mathrm{d} \mathcal{V}$,

where $R_{b}$ is the radius of the body nose. We notice that the factor $\cos \chi_{1}$ is not included in Eq. (70), partly ignoring the influence of the shock inclination. The saturation effect in the limit of large $B$ has been mentioned by other authors in the context of MHD power generation with a Faraday-type generator [32].

\subsubsection{Bow shock}

As discussed in our reference paper [3], an experiment could be devised to check phenomena in the shock layer behind the shock formed ahead of a reentry blunt body of RAM-C type moving at a hypersonic speed. In the present situation with Hall effect, an analytical model would be needed in order to yield a theoretical expression of the stand-off distance.

Indeed, a valuable semi-analytical model has been published some time ago [40], but the numerical applications have been run with argon as working gas. Thus the conclusions concerning the shock standoff distance (Figure 7 in [40]) cannot be easily transposed to air. In particular, the shock distance is not necessarily larger when the vehicle wall is electrically insulating, as compared with the conducting case, a behavior that is apparently not confirmed by recent numerical experiments with air [36]. On the other hand, laboratory experiments, confirmed by numerical simulations, show that the shock distance is approximately twice as large in air than in Argon at Mach 9.2 and 8.8 [44].

Other laboratory experiments, carried on a sphere-cylinder reentry body equipped with a 1T-electromagnet in a hypersonic tunnel of the CIRA SCIROCCO facility, rebuilt with numerical codes, provide valuable material for partial assessment of our model at Mach 10 [12]. In one of their experiments, the authors consider a scalar conductivity of $7 \mathrm{~S} \mathrm{~m}^{-1}$, consistent with the value of approximately $16 \mathrm{~S} \mathrm{~m}^{-1}$ estimated from our fig. $9 b$ at $M_{1}=10$. The azimuthal current $J_{z}$ in the shock layer, close to the shock, amounts to a few $100 \mathrm{~A} \mathrm{~m}^{-2}$, while we can estimate it as approximately $100 \mathrm{~A} \mathrm{~m}^{-2}$ from our fig. $10 \mathrm{~b}$ at $M_{1}=10$. An interesting feature reported in that work shows that the difference of pressure on the body wall, estimated with a scalar or tensor conductivity, is negligible: this property is consistent with our remark in Sect. 3.2 above, stating that, in the presence of Hall effect, the influence of the magnetic field on $J_{z}$ (Fig. 10b) and $w_{2}$ (Fig. 11b) becomes sensitive for $M_{1} \geq 10$.

Researchers involved in OREX imagined to equip the body nose with a Hall-type generator in order to harvest electric power [21]. Results are obtained with a velocity of $6223 \mathrm{~m} \mathrm{~s}^{-1}\left(M_{1} \approx 20\right)$ and a nominal magnetic field of $0.5 \mathrm{~T}$ at an altitude of $64 \mathrm{~km}$. They show ordinary electric conductivities in the range 120 to $180 \mathrm{~S} \mathrm{~m}^{-1}$, consistently with our values $\left(\sigma_{e} \approx 220 \mathrm{~S} \mathrm{~m}^{-1}\right)$.

\subsubsection{Equilibrium vs non-equilibrium}

Turning to a crucial point of the present model, we consider that the air plasma inside the shock is in thermochemical equilibrium, although estimations of the Damköhler number $N_{D}$ reported in our previous paper [3] and calculated with a specific methodology [8], suggest that chemical equilibrium is probably realized at an altitude of $40 \mathrm{~km}\left(N_{D}=10.3\right)$, but that it is not at $60 \mathrm{~km}\left(N_{D}=0.039\right)$. Actually, in the transition region we consider (altitude $\approx 65 \mathrm{~km}$, velocity $\approx 7 \mathrm{~km} \mathrm{~s}^{-1}$ ), this calculation confirms chemical non-equilibrium, but not thermal non-equilibrium, as can be checked from a standard work dealing with a $30.5 \mathrm{~cm}$-radius sphere (Fig. 1 in ref. [24]).

Two models of blunt-body problem with non-equilibrium (NE) air plasma provide electron densities $n_{e}$ behind a RAM-C shock on the stagnation line at three altitudes $(61 \mathrm{~km}, 71$ and $81 \mathrm{~km})$, with three different Mach numbers (23.9, 25.9 and 28.3 respectively), for the first one [9], and at two altitudes (61 km and $71 \mathrm{~km})$, with two Mach numbers (24 and 26 respectively), for the second one [17]. The comparison of these data, with a plot of our present model using the power law, at $M=23$ for a normal shock $\left(\chi_{1}=0^{\circ} ; \chi_{b}=180^{\circ}\right)$, shows that the present model is located in between these two reference results in the range 60 to $70 \mathrm{~km}$ (Fig. 15). Results obtained with the simplified 5-species $\left(\mathrm{N}_{2}, \mathrm{O}_{2}, \mathrm{~N}, \mathrm{O}, \mathrm{NO}^{+}\right.$, plus electrons) plasma model in equilibrium we used in [3], with standard kinetic constants [37], are shown for comparison, and they lie below the other models. 
We have taken frozen chemistry into account in our methodology by introducing a parameter $\zeta$, Eq. (30) (Sect. 2.4), that ensures that the ionization depth, connected to $\mathscr{L}_{\sigma}$, is much larger than the shock thickness represented by $\mathscr{L}_{\rho} \quad(\zeta=10)$. In order to improve this model in future work, we propose a discussion in the prospective Sect.4, based on equations governing the concentrations of plasma species, that we derive in Appendix 6. According to ref. [24], a minimal 5-species model $\left(\mathrm{N}_{2}, \mathrm{O}_{2}, \mathrm{~N}, \mathrm{O}, \mathrm{NO}^{+}\right)$could be sufficient to describe the plasma at an altitude of $65 \mathrm{~km}$ and a velocity of $7 \mathrm{~km} \mathrm{~s}^{-1}$, with three chemical reactions and their reciprocals.

\subsubsection{Hall parameters}

Since these are key parameters governing the Hall effect, they deserve special attention. Using Eqs. (5a) and (6), we can recast $\beta_{e}$ and $\beta_{i}$ as:

$\beta_{e}=\frac{\sigma_{e}}{n_{e} q_{e}} B, \quad \beta_{i}=C \beta_{e}, \quad C=\frac{q_{i}}{q_{e}} \frac{m_{e}}{m_{i}} \frac{v_{e}}{v_{i}}$.

Eq. (72a) shows that the electron parameter $\beta_{e}$ depends on the ratio $\sigma_{e} / n_{e}$. We know from the discussion of Sect. 3.4.3 that $n_{e}$ is probably well estimated with our semi-equilibrium model. As for the electric conductivity, Fig. $16 b$ in our previous work [3] shows that the power law model may overestimate $\sigma_{e}$ by a factor 2, compared to an equilibrium model. The factor $C$ can be estimated with $\mathrm{NO}^{+}\left(q_{i}=q_{e}, m_{e} / m_{i}=1.8 \times 10^{-5}\right)$ as main ion species and the simplified 5-species model already used in [3], yielding the ratio of collision frequencies $v_{e} / v_{i}=129$, and the factor $C \approx 2.3 \times 10^{-3}$.

OREX modelers included the ion slip factor in their CFD simulation of the Hall effect [21]. The range of the Hall parameters and slip factor they obtain along a cross line in the shock layer $\left(\beta_{e} \approx 12\right.$ to $23 ; \beta_{i} \approx 0.03$ to 0.04 ; $s \approx 0.36$ to 0.92$)$, can be compared with our own estimates in the shock front $\left(\beta_{e} \approx 50 ; s \approx 6,3\right)$ in the same conditions (Fig. $12 a$ and fig. 12b). Although our slip factor seems larger by an order of magnitude, the ratio $\beta_{i}$ $/ \beta_{e}\left(\approx 2 \times 10^{-3}\right)$ is about the same as our constant $C$ estimated above. Furthermore, a radial profile of $\beta_{e}$ in the shock layer exhibits a sharp increase in the vicinity of the shock front [20].

\section{Conclusion and prospect}

The present situation is paradoxical, because the Hall effect arises from the fact that the magnetic field dominates over collisions, and, under certain boundary conditions imposed on the electric current density (as a solution of the equation of conservation of electric charge), it finally counterbalances MHD processes, even in the absence of dissipation (viscous or Ohmic). In the case of the shock front, there is no current ahead of the shock and thus the current normal to the shock vanishes.

This is confirmed by the fact that the usual way of defining the interaction parameter, or Stuart number, $S$, is misleading when the Hall effect is developed, because $S$ would increase without limit as the magnetic field $B$ increases, while the Laplace force becomes saturated. The present situation can be related to the problem raised by other authors about the estimation of the magnetic Reynolds number when the induced magnetic field is not taken into account [19].

In the blunt-body problem with an electrically conducting wall, the current loops in the shock layer enter the body wall, so that the shock is no more efficiently repelled by MHD forces. The Hall effect acts as a moderating phenomenon, and it would finally produce a saturation of the MHD forces if the magnetic field could be increased significantly (above $10 \mathrm{~T}$ ). Conversely, the Hall effect may be used constructively to generate power if the vehicle is equipped with a Hall-type generator, although the performances are also lowered by the ion slip factor $s$, up to $22 \%$ of the power peak output at an altitude of $64 \mathrm{~km} \mathrm{[21].} \mathrm{Another} \mathrm{outstanding} \mathrm{feature} \mathrm{shows}$ that certain parameters, still sensitive to the magnetic field in presence of Hall effect $\left(J_{z 2}, w_{2}\right)$, exhibit a clear $B$ dependence if $M_{1} \geq 10$, in agreement with laboratory experiments at Mach 10 [12].

In our basic analysis without Hall effect [3], the importance of the MHD interaction in the shock front itself was shown to amount to $6 \%$ of the aerodynamic interaction, whereas it amounts to $60 \%$ in the shock layer. The jump equations provided boundary conditions for finding analytic flow solutions behind the shock. Ideally, analytic solutions expressed by means of stream functions would give more insight in the flow topology. Details of the general procedure, using our jump relations as boundary conditions at the shock, can be found in [3].

Lastly, according to the Damköhler number estimated in our previous work [3], we know that chemical nonequilibrium is likely to arise in the shock, and this contribution is modeled by means of the parameter $\zeta$ introduced in Eq. (30). On the other hand, according to an 11-species model [24], the assumption of thermal equilibrium may be still valid at an altitude of $65 \mathrm{~km}$ and Mach 23. 
In Appendix 6 below, we sketch a procedure improving our methodology by taking into account a deviation from chemical equilibrium in the equations of conservation of individual species in our 5-species model $\left(\mathrm{N}_{2}, \mathrm{O}_{2}\right.$, $\mathrm{N}, \mathrm{O}, \mathrm{NO}^{+}$). Defining the four Damköhler numbers:

$\left\{\begin{array}{l}N_{D M_{1}}=\frac{k_{f 1} C_{M_{1}} \mathscr{L}_{M \rho}}{u} \\ N_{D M_{2}}=\frac{k_{f 2} C_{M_{2}} \mathscr{L}_{M_{\rho} \rho}}{u}\end{array}\left\{\begin{array}{l}N_{D \mathrm{~N}_{2}}=\frac{2 k_{b 3}\left(C_{\mathrm{N}_{2}}\right)_{1} \mathscr{L}_{\rho M} u_{1}}{u^{2}} \\ N_{D \mathrm{O}_{2}}=\frac{2 k_{b 3}\left(C_{\mathrm{O}_{2}}\right)_{1} \mathscr{L}_{\rho M} u_{1}}{u^{2}}\end{array}\right.\right.$

and the characteristic electron concentration built on the normal velocity $u$ :

$N_{e u}=\frac{u}{2 k_{b 3} \mathcal{L}_{\rho \mathbb{l l}}}$,

we recast the coupled quadratic Eqs. (134) and (136) as:

$\left\{\begin{array}{l}2 \frac{C_{\mathrm{N}}^{2}}{K_{1}\left(1-\frac{1}{N_{D M_{1}}}\right)}+\left(1+\frac{K_{3} C_{\mathrm{O}}}{N_{e}}\right) C_{\mathrm{N}}-2\left(1-\frac{1}{N_{D \mathrm{~N}_{2}}}\right) \frac{u_{1}}{u}\left(C_{\mathrm{N}_{2}}\right)_{1}=0 \\ 2 \frac{C_{\mathrm{O}}^{2}}{K_{2}\left(1-\frac{1}{N_{D M_{2}}}\right)}+\left(1+\frac{K_{3} C_{\mathrm{N}}}{N_{e}}\right) C_{\mathrm{O}}-2\left(1+\frac{1}{N_{D \mathrm{O}_{2}}}\right) \frac{u_{1}}{u}\left(C_{\mathrm{O}_{2}}\right)_{1}=0\end{array}\right.$

and:

$N_{e}=\sqrt{K_{3} C_{\mathrm{N}} C_{\mathrm{O}}+N_{e u}^{2}}+N_{e u}$

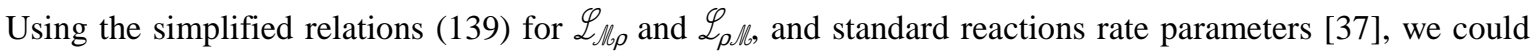
estimate the above Damköhler numbers midway across the shock $\left(u=\left(u_{1} u_{2}\right)^{1 / 2}\right)$, without (Table 3$)$ and with (Table 4) Hall effect, and thus we obtained the following results.

Concerning the third terms in Eqs. (75), the $\mathrm{N}_{2}$ - and $\mathrm{O}_{2}$-components of the plasma are both at equilibrium $\left(N_{D \mathrm{~N} 2} \gg 1 ; N_{D \mathrm{O} 2} \gg 1\right)$, and therefore these two terms will reduce to the source terms multiplied by $u_{1} / u$, i.e. by the density ratio $\rho / \rho_{1}$ (conservation of total mass, Eq. (11)). In contrast, the Damköhler numbers $N_{D \mathrm{M} 1}$ and $N_{D \mathrm{M} 2}$, estimated with $M_{1}=\mathrm{N}_{2}$ and $M_{2}=\mathrm{O}_{2}$, suggest that the $\mathrm{N}_{2}$ - and $\mathrm{O}_{2}$-components governing the quadratic terms in Eqs. (75) are frozen $\left(N_{D M 1}<<1 ; N_{D M 2}<<1\right)$ [23]

Therefore, considering the Damköhler numbers that can be naturally defined from our local non-dimensional equations, it seems that some contributions reflect equilibrium conditions and other contributions nonequilibrium. Moreover, the decrease of $N_{\mathrm{DO} 2}$ suggests that the Hall effect tends to displace the $\mathrm{O}_{2}$-component toward non-equilibrium, while the $\mathrm{N}_{2}$-component keeps stable (Table 4).

By substituting the equilibrium value (138) of $N_{e}^{*}$ in (76), we obtain an estimate of the value $N_{e}{ }^{* *}$ in nonequilibrium:

$N_{e}^{* *}=\sqrt{N_{e}^{*}+N_{e u}^{2}}+N_{e u}$.

The electron concentration $N_{e}$ (in mol $\mathrm{m}^{-3}$ ) and the electron density $n_{e}$ (in $\mathrm{m}^{-3}$ ) are related by the Avogadro number $\mathcal{N}_{A}\left(n_{e}=\mathcal{N}_{A} N_{e}\right)$. The plot of $n_{e}{ }^{* *}$ derived from (77) for a normal shock $\left(\chi_{1}=0^{\circ} ; \chi_{b}=180^{\circ}\right)$ shows that it is in very good agreement with the electron density $n_{e}$ derived from the power-law conductivity (Fig. 15).

A further refinement would consist in integrating the differential system Eqs. (120), (121), (122) between $x_{1}$ (shock front) and $x_{2}$ (shock backside) in order to get the jumps of species concentrations, and then solving the complete algebraic system composed of Eqs. (21a-e) and the integrated form of Eqs. (120), (121), and (122).

Acknowledgements This work was supported by funding from ONERA. The author is indebted to two anonymous referees for valuables comments and to the remarks of one of them drawing the author's attention to discuss equilibrium assumptions and experimental validation. 


\section{Appendix 1}

This appendix is devoted to the derivation of the following basic relations to be used for the calculation of the integrals of the differential system (10) in Appendix 2:

$$
\left\{\begin{array}{l}
K \sigma_{P}=\mathscr{L}_{\sigma} \frac{\mathrm{d}\left(K \sigma_{P}\right)}{\mathrm{d} x} \\
K \sigma_{H}=\mathscr{L}_{\sigma} \frac{\mathrm{d}\left(K \sigma_{H}\right)}{\mathrm{d} x} \\
N \sigma_{P}=\mathscr{L}_{\sigma} \frac{\mathrm{d}\left(N \sigma_{P}\right)}{\mathrm{d} x}
\end{array}\right.
$$

Using the relations (16), (17) and (19), we can develop the following calculations:

1)

$$
\begin{aligned}
\frac{\mathrm{d}\left(K \sigma_{P}\right)}{\mathrm{d} x} & =\sigma_{P} \frac{\mathrm{d}}{\mathrm{d} x}\left(\frac{\sigma_{e} \cos ^{2} \chi_{b}}{D}\right)+K \frac{\mathrm{d} \sigma_{P}}{\mathrm{~d} x} \\
& =\frac{\sigma_{P} \cos ^{2} \chi_{b}}{D} \frac{\mathrm{d} \sigma_{e}}{\mathrm{~d} x}-\frac{\sigma_{P} \sigma_{e} \cos ^{2} \chi_{b}}{D^{2}}\left(\frac{\mathrm{d} \sigma_{e}}{\mathrm{~d} x} \cos ^{2} \chi_{b}+\frac{\mathrm{d} \sigma_{P}}{\mathrm{~d} x} \sin ^{2} \chi_{b}\right)+K \frac{\mathrm{d} \sigma_{P}}{\mathrm{~d} x} \\
& =\frac{\sigma_{P} \sigma_{e} \cos ^{2} \chi_{b}}{D \mathscr{L}_{\sigma}}-\frac{\sigma_{P} \sigma_{e} \cos ^{2} \chi_{b}}{D^{2}}\left(\frac{\sigma_{e}}{\mathscr{L}_{\sigma}} \cos ^{2} \chi_{b}+\frac{\sigma_{P}}{\mathscr{L}_{\sigma}} \sin ^{2} \chi_{b}\right)+K \frac{\sigma_{P}}{\mathscr{L}_{\sigma}} \\
& =\left(2 K-\frac{\sigma_{e} \cos ^{2} \chi_{b}}{D}\right) \frac{\sigma_{P}}{\mathscr{L}_{\sigma}} \\
& =\frac{K \sigma_{P}}{\mathscr{L}_{\sigma}}
\end{aligned}
$$

2)

$$
\begin{aligned}
\frac{\mathrm{d}\left(K \sigma_{H}\right)}{\mathrm{d} x} & =\sigma_{H} \frac{\mathrm{d}}{\mathrm{d} x}\left(\frac{\sigma_{e} \cos ^{2} \chi_{b}}{D}\right)+K \frac{\mathrm{d} \sigma_{H}}{\mathrm{~d} x} \\
& =\frac{\sigma_{H} \cos ^{2} \chi_{b}}{D} \frac{\mathrm{d} \sigma_{e}}{\mathrm{~d} x}-\frac{\sigma_{H} \sigma_{e} \cos ^{2} \chi_{b}}{D^{2}}\left(\frac{\mathrm{d} \sigma_{e}}{\mathrm{~d} x} \cos ^{2} \chi_{b}+\frac{\mathrm{d} \sigma_{P}}{\mathrm{~d} x} \sin ^{2} \chi_{b}\right)+K \frac{\mathrm{d} \sigma_{H}}{\mathrm{~d} x} \\
& =\frac{\sigma_{H} \sigma_{e} \cos ^{2} \chi_{b}}{D \mathscr{L}_{\sigma}}-\frac{\sigma_{H} \sigma_{e} \cos ^{2} \chi_{b}}{D^{2}}\left(\frac{\sigma_{e}}{\mathscr{L}_{\sigma}} \cos ^{2} \chi_{b}+\frac{\sigma_{P}}{\mathscr{L}_{\sigma}} \sin ^{2} \chi_{b}\right)+K \frac{\sigma_{H}}{\mathscr{L}_{\sigma}} \\
& =\left(2 K-\frac{\sigma_{e} \cos ^{2} \chi_{b}}{D}\right) \frac{\sigma_{H}}{\mathscr{L}_{\sigma}} \\
& =\frac{K \sigma_{H}}{\mathscr{L}_{\sigma}}
\end{aligned}
$$

3)

$$
\begin{aligned}
\frac{\mathrm{d}\left(N \sigma_{P}\right)}{\mathrm{d} x} & =\sigma_{P} \frac{\mathrm{d}}{\mathrm{d} x}\left(\frac{\sigma_{e} \cos ^{2} \chi_{b}+\sigma_{C} \sin ^{2} \chi_{b}}{D}\right)+N \frac{\mathrm{d} \sigma_{P}}{\mathrm{~d} x} \\
& =\frac{\sigma_{P}}{D}\left(\frac{\mathrm{d} \sigma_{e}}{\mathrm{~d} x} \cos ^{2} \chi_{b}+\frac{\mathrm{d} \sigma_{C}}{\mathrm{~d} x} \sin ^{2} \chi_{b}\right) \\
& -\frac{\sigma_{P}}{D^{2}}\left(\sigma_{e} \cos ^{2} \chi_{b}+\sigma_{C} \sin ^{2} \chi_{b}\right)\left(\frac{\mathrm{d} \sigma_{e}}{\mathrm{~d} x} \cos ^{2} \chi_{b}+\frac{\mathrm{d} \sigma_{P}}{\mathrm{~d} x} \sin ^{2} \chi_{b}\right)+N \frac{\mathrm{d} \sigma_{P}}{\mathrm{~d} x} \\
& =\frac{\sigma_{P}}{D}\left(\frac{\sigma_{e}}{\mathscr{L}_{\sigma}} \cos ^{2} \chi_{b}+\frac{\sigma_{C}}{\mathscr{L}_{\sigma}} \sin ^{2} \chi_{b}\right) \\
& -\frac{\sigma_{P}}{D^{2}}\left(\sigma_{e} \cos ^{2} \chi_{b}+\sigma_{C} \sin ^{2} \chi_{b}\right)\left(\frac{\sigma_{e}}{\mathscr{L}_{\sigma}} \cos ^{2} \chi_{b}+\frac{\sigma_{P}}{\mathscr{L}_{\sigma}} \sin ^{2} \chi_{b}\right)+N \frac{\sigma_{P}}{\mathscr{L}_{\sigma}} \\
& =\frac{N \sigma_{P}}{\mathscr{L}_{\sigma}}
\end{aligned}
$$

which show that the products $K \sigma_{P}, K \sigma_{H}$ and $N \sigma_{P}$ satisfy the same relations (16) and (17), like $\sigma_{e}, \sigma_{p}$ and $\sigma_{H}$. 


\section{Appendix 2}

This appendix is devoted to the derivation of the relations (20), to be used for integration of system (10). As shown in Appendix 1, the products $K \sigma_{P}, K \sigma_{H}$ et $L \sigma_{P}$ satisfy the same type of relation as $\sigma_{e}, \sigma_{p}$ and $\sigma_{H}$, namely Eqs. (78). By integrating the integrals by parts, and using the above relations (78), we obtain successively:

1)

$$
\begin{aligned}
\int_{x_{1}}^{x_{2}} \frac{\mathrm{d}}{\mathrm{d} x}\left(N \sigma_{P} u\right) \mathrm{d} x & =\int_{x_{1}}^{x_{2}} N \sigma_{P} \frac{\mathrm{d} u}{\mathrm{~d} x} \mathrm{~d} x+\int_{x_{1}}^{x_{2}} u \frac{\mathrm{d}\left(N \sigma_{P}\right)}{\mathrm{d} x} \mathrm{~d} x \\
& =-\frac{1}{\mathscr{L}_{\rho}} \int_{x_{1}}^{x_{2}} N \sigma_{P} u \mathrm{~d} x+\frac{1}{\mathscr{L}_{\sigma}} \int_{x_{1}}^{x_{2}} N \sigma_{P} u \mathrm{~d} x \\
& =\frac{1}{\mathscr{L}_{\rho \sigma}} \int_{x_{1}}^{x_{2}} N \sigma_{P} u \mathrm{~d} x
\end{aligned}
$$

2)

$$
\begin{aligned}
\int_{x_{1}}^{x_{2}} \frac{\mathrm{d}}{\mathrm{d} x}\left(N \sigma_{P} v\right) \mathrm{d} x & =\int_{x_{1}}^{x_{2}} N \sigma_{P} \frac{\mathrm{d} v}{\mathrm{~d} x} \mathrm{~d} x+\int_{x_{1}}^{x_{2}} v \frac{\mathrm{d}\left(N \sigma_{P}\right)}{\mathrm{d} x} \mathrm{~d} x \\
& =\frac{1}{\mathscr{L}_{v}} \int_{x_{1}}^{x_{2}} N \sigma_{P} v \mathrm{~d} x+\frac{1}{\mathscr{L}_{\sigma}} \int_{x_{1}}^{x_{2}} N \sigma_{P} v \mathrm{~d} x \\
& =\frac{1}{\mathscr{L}_{v \sigma}} \int_{x_{1}}^{x_{2}} N \sigma_{P} v \mathrm{~d} x
\end{aligned}
$$

3)

$$
\begin{aligned}
\int_{x_{1}}^{x_{2}} \frac{\mathrm{d}}{\mathrm{d} x}\left(K \sigma_{H} w\right) \mathrm{d} x & =\int_{x_{1}}^{x_{2}} K \sigma_{H} \frac{\mathrm{d} w}{\mathrm{~d} x} \mathrm{~d} x+\int_{x_{1}}^{x_{2}} w \frac{\mathrm{d}\left(K \sigma_{H}\right)}{\mathrm{d} x} \mathrm{~d} x \\
& =\frac{1}{\mathscr{L}_{w}} \int_{x_{1}}^{x_{2}} K \sigma_{H} w \mathrm{~d} x+\frac{1}{\mathscr{L}_{\sigma}} \int_{x_{1}}^{x_{2}} K \sigma_{H} w \mathrm{~d} x \\
& =\frac{1}{\mathscr{L}_{w \sigma}} \int_{x_{1}}^{x_{2}} K \sigma_{H} w \mathrm{~d} x
\end{aligned}
$$

4)

$$
\begin{aligned}
\int_{x_{1}}^{x_{2}} \frac{\mathrm{d}}{\mathrm{d} x}\left(K \sigma_{H} u\right) \mathrm{d} x & =\int_{x_{1}}^{x_{2}} K \sigma_{H} \frac{\mathrm{d} u}{\mathrm{~d} x} \mathrm{~d} x+\int_{x_{1}}^{x_{2}} u \frac{\mathrm{d}\left(K \sigma_{H}\right)}{\mathrm{d} x} \mathrm{~d} x \\
& =-\frac{1}{\mathscr{L}_{\rho}} \int_{x_{1}}^{x_{2}} K \sigma_{H} u \mathrm{~d} x+\frac{1}{\mathscr{L}_{\sigma}} \int_{x_{1}}^{x_{2}} K \sigma_{H} u \mathrm{~d} x \\
& =\frac{1}{\mathscr{L}_{\rho \sigma}} \int_{x_{1}}^{x_{2}} K \sigma_{H} u \mathrm{~d} x
\end{aligned}
$$

5)

$$
\begin{aligned}
\int_{x_{1}}^{x_{2}} \frac{\mathrm{d}}{\mathrm{d} x}\left(K \sigma_{P} w\right) \mathrm{d} x & =\int_{x_{1}}^{x_{2}} K \sigma_{P} \frac{\mathrm{d} w}{\mathrm{~d} x} \mathrm{~d} x+\int_{x_{1}}^{x_{2}} w \frac{\mathrm{d}\left(K \sigma_{P}\right)}{\mathrm{d} x} \mathrm{~d} x \\
& =\frac{1}{\mathscr{L}_{w}} \int_{x_{1}}^{x_{2}} K \sigma_{P} w \mathrm{~d} x+\frac{1}{\mathscr{L}_{\sigma}} \int_{x_{1}}^{x_{2}} K \sigma_{P} w \mathrm{~d} x \\
& =\frac{1}{\mathscr{L}_{w \sigma}} \int_{x_{1}}^{x_{2}} K \sigma_{P} w \mathrm{~d} x
\end{aligned}
$$

where, like in the situation without Hall effect, we have defined the compound length scales $\mathscr{L}_{\rho \sigma}, \mathscr{L}_{v \sigma}$ and $\mathscr{L}_{w \sigma}$ :

$$
\left\{\begin{array}{l}
\frac{1}{\mathscr{L}_{\rho \sigma}}=\frac{1}{\mathscr{L}_{\sigma}}-\frac{1}{\mathscr{L}_{\rho}}, \\
\frac{1}{\mathscr{L}_{v \sigma}}=\frac{1}{\mathscr{L}_{\sigma}}+\frac{1}{\mathscr{L}_{v}}, \\
\frac{1}{\mathscr{L}_{w \sigma}}=\frac{1}{\mathscr{L}_{\sigma}}+\frac{1}{\mathscr{L}_{w}} .
\end{array}\right.
$$

Note that $\mathscr{L}_{\rho \sigma}$ can be positive or negative. 


\section{Appendix 3}

This appendix details the main steps in solving the algebraic system (22) for the five unknown jumps $[p],[\rho]$, $[u],[v],[w]$, or, equivalently, $p_{2}, \rho_{2}, u_{2}, v_{2}, w_{2}$, since the upstream values $p_{1}, \rho_{1}, u_{1}, v_{1}, w_{1}$, are known. The development of the quantities in brackets in Eqs. $(22 b-e)$ yields:

$$
\left\{\begin{array}{l}
u_{2}-u_{1}+\frac{p_{2}-p_{1}}{m}=-\left(\frac{N_{2} \sigma_{P 2} \mathscr{L}_{\rho \sigma} \sin \chi_{b}}{m} u_{2}-\frac{N_{2} \sigma_{P 2} \mathscr{L}_{v \sigma} \cos \chi_{b}}{m} v_{2}+\frac{K_{2} \sigma_{H 2} \mathscr{L}_{w \sigma} \cos ^{2} \chi_{b}}{m} w_{2}\right) B^{2} \sin \chi_{b} \\
v_{2}-v_{1}=\left(\frac{N_{2} \sigma_{P 2} \mathscr{L}_{\rho \sigma} \sin \chi_{b}}{m} u_{2}-\frac{N_{2} \sigma_{P 2} \mathscr{L}_{v \sigma} \cos \chi_{b}}{m} v_{2}+\frac{K_{2} \sigma_{H 2} \mathscr{L}_{w \sigma} \cos ^{2} \chi_{b}}{m} w_{2}\right) B^{2} \cos \chi_{b} \\
w_{2}=\left(\frac{K_{2} \sigma_{H 2} \mathscr{L}_{\rho \sigma} \sin \chi_{b}}{m} u_{2}-\frac{K_{2} \sigma_{P 2} \mathscr{L}_{w \sigma}}{m} w_{2}\right) B^{2} \cos ^{2} \chi_{b} \\
\left(\frac{\gamma_{2}}{\gamma_{2}-1} \frac{p_{2}}{\rho_{2}}-\frac{\gamma_{1}}{\gamma_{1}-1} \frac{p_{1}}{\rho_{1}}\right)+\frac{u_{2}^{2}-u_{1}^{2}}{2}+\frac{v_{2}^{2}-v_{1}^{2}}{2}+\frac{w_{2}^{2}}{2}=0
\end{array}\right.
$$

After defining the microscopic interaction parameters $S_{\rho \sigma}, S_{v \sigma}, S_{w \sigma}$ respectively built on the length scales $\mathscr{L}_{\rho \sigma}$, $\mathscr{L}_{v \sigma} \mathscr{L}_{w \sigma}\left(\right.$ Eqs. (87)) and $\sigma_{e 2}, B, m$ :

$$
S_{\rho \sigma}=\frac{\sigma_{e 2} B^{2} \mathscr{L}_{\rho \sigma}}{m}, \quad S_{v \sigma}=\frac{\sigma_{e 2} B^{2} \mathscr{L}_{v \sigma}}{m}, \quad S_{w \sigma}=\frac{\sigma_{e 2} B^{2} \mathscr{L}_{w \sigma}}{m},
$$

then denoting electric conductivities normalized to $\sigma_{e 2}$ with a tilde $\sim$ (Eqs. (26)) and reorganizing terms, the subset of Eqs. $(88 a, b, c)$ becomes:

$$
\left\{\begin{array}{l}
\left(1+N_{2} \tilde{\sigma}_{P 2} S_{\rho \sigma} \sin ^{2} \chi_{b}\right) u_{2}-N_{2} \tilde{\sigma}_{P 2} S_{v \sigma} \sin \chi_{b} \cos \chi_{b} v_{2}+K_{2} \tilde{\sigma}_{H 2} S_{w \sigma} \sin \chi_{b} \cos ^{2} \chi_{b} w_{2}=u_{1}+\frac{p_{1}-p_{2}}{m} \\
-N_{2} \tilde{\sigma}_{P 2} S_{\rho \sigma} \sin \chi_{b} \cos \chi_{b} u_{2}+\left(1+N_{2} \tilde{\sigma}_{P 2} S_{v \sigma} \cos ^{2} \chi_{b}\right) v_{2}-K_{2} \tilde{\sigma}_{H 2} S_{w \sigma} \cos ^{3} \chi_{b} w_{2}=v_{1} \\
-K_{2} \tilde{\sigma}_{H 2} S_{\rho \sigma} \sin \chi_{b} \cos ^{2} \chi_{b} u_{2}+\left(1+K_{2} \tilde{\sigma}_{P 2} S_{w \sigma} \cos ^{2} \chi_{b}\right) w_{2}=0
\end{array}\right.
$$

Now, we first extract $w_{2}$ from $(90 c)$ and then insert it into $(90 a, b)$ to obtain a Cramer system for $u_{2}, v_{2}$, whose determinant can be written:

$D_{2}=1+\tilde{\sigma}_{P 2} N_{2}\left(S_{\rho \sigma} \sin ^{2} \chi_{b}+S_{v \sigma} \cos ^{2} \chi_{b}\right)+\frac{K_{2}^{2} \tilde{\sigma}_{H 2}^{2} S_{\rho \sigma} S_{w \sigma} \sin ^{2} \chi_{b} \cos ^{4} \chi_{b}}{1+K_{2} \tilde{\sigma}_{P 2} S_{w \sigma} \cos ^{2} \chi_{b}}$,

and, therefore, the velocity components are found as:

$$
\left\{\begin{array}{l}
u_{2}=a\left(u_{1}+\frac{p_{1}-p_{2}}{m}\right)+b v_{1} \\
v_{2}=c\left(u_{1}+\frac{p_{1}-p_{2}}{m}\right)+d v_{1} \\
w_{2}=f u_{2}
\end{array}\right.
$$

with the four coefficients:

$$
\left\{\begin{array}{l}
a=\frac{1+N_{2} \tilde{\sigma}_{P 2} S_{v \sigma} \cos ^{2} \chi_{b}}{D_{2}} \\
b=\frac{N_{2} \tilde{\sigma}_{P 2} S_{v \sigma} \sin \chi_{b} \cos \chi_{b}}{D_{2}} \\
c=\left(N_{2} \tilde{\sigma}_{P 2}+\frac{K_{2}^{2} \tilde{\sigma}_{H 2}^{2} S_{w \sigma} \cos ^{4} \chi_{b}}{1+K_{2} \tilde{\sigma}_{P 2} S_{w \sigma} \cos ^{2} \chi_{b}}\right) \frac{S_{\rho \sigma} \sin \chi_{b} \cos \chi_{b}}{D_{2}} \\
d=\frac{1}{D_{2}}\left(1+N_{2} \tilde{\sigma}_{P 2} S_{\rho \sigma} \sin ^{2} \chi_{b}+\frac{K_{2}^{2} \tilde{\sigma}_{H 2}^{2} S_{\rho \sigma} S_{w \sigma} \sin ^{2} \chi_{b} \cos ^{4} \chi_{b}}{1+K_{2} \tilde{\sigma}_{P 2} S_{w \sigma} \cos ^{2} \chi_{b}}\right)
\end{array}\right.
$$


and:

$f=\frac{K_{2} \tilde{\sigma}_{H 2} S_{\rho \sigma} \sin \chi_{b} \cos ^{2} \chi_{b}}{1+K_{2} \tilde{\sigma}_{P 2} S_{w \sigma} \cos ^{2} \chi_{b}}$.

Using the relations (33), the ambient physical conditions of Table 2 and downstream values of Table 4, we obtain the values of the product $S_{\rho \sigma} S_{w \sigma}$. The coefficients $K$ and $N$ defined by (12a,b) being of order unity, the above calculation justifies neglecting the second-order quantities proportional to $S_{\rho \sigma} S_{w \sigma}$, and it follows thereupon that the four coefficients $a, b, c, d$ can be written in the simplified form of Eqs. (24).

Then, inserting the expressions of the velocity components, Eqs. $(92 a, b, c)$, into the energy equation, Eq. $(88 d)$, using the incident Mach number:

$M_{1}=\frac{V_{1}}{c_{s 1}}$,

sound speed:

$c_{s 1}=\sqrt{\gamma_{1} \frac{p_{1}}{\rho_{1}}}$

and velocity components:

$\left\{\begin{array}{l}u_{1}=V_{1} \cos \chi_{1} \\ v_{1}=V_{1} \sin \chi_{1}\end{array}\right.$

we finally obtain the quadratic equations satisfied by the pressure, Eq. (34), and the mass density, Eq. (37).

\section{Appendix 4}

This appendix is devoted to the derivation of the local Laplace force vector $\boldsymbol{F}$ and to the calculation of its magnitude in the limit of infinite $B$. Since there is no current in front of the shock, the condition of current conservation $\left(\partial J_{x} / \partial x=0\right)$ implies that $J_{x}=0$ everywhere. Thus, from the current density along $x$, Eq. $(4 a)$, we obtain the electric field $E_{x 2}$ :

$E_{x 2}=\frac{\sigma_{H 2}\left(-u_{2} \sin \chi_{b}+v_{2} \cos \chi_{b}\right)+\sigma_{P 2} w_{2}}{\sigma_{e 2} \cos ^{2} \chi_{b}+\sigma_{P 2} \sin ^{2} \chi_{b}} B \sin \chi_{b}$.

Therefore, the non-zero components of the electric current density can be recast as:

$\left\{\begin{array}{l}J_{y 2}=\frac{\sigma_{H 2}\left(-u_{2} \sin \chi_{b}+v_{2} \cos \chi_{b}\right)+\sigma_{P 2} w_{2}}{\cos \chi_{b}} K_{2} B \\ J_{z 2}=\left\{\sigma_{P 2} N_{2}\left(u_{2} \sin \chi_{b}-v_{2} \cos \chi_{b}\right)-\sigma_{H 2} K_{2} w_{2}\right\} B\end{array}\right.$

Making use of the relations (27), we obtain:

$\left\{\begin{array}{l}J_{y 2}=\frac{\left(f \sigma_{P 2}-\sigma_{H 2} \sin \chi_{b}\right) \varepsilon u_{1}+\eta \sigma_{H 2} v_{1} \cos \chi_{b}}{\cos \chi_{b}} K_{2} B \\ J_{z 2}=\left\{\left(\sigma_{P 2} N_{2} \sin \chi_{b}-f \sigma_{H 2} K_{2}\right) \varepsilon u_{1}-\eta \sigma_{P 2} N_{2} v_{1} \cos \chi_{b}\right\}_{B}\end{array}\right.$

The components of the Laplace force vector, obtained from the cross product of current and magnetic field, are such that:

$\boldsymbol{J}\left|\begin{array}{l}0 \\
J_{y 2} \\
J_{z 2}\end{array} \quad \times \boldsymbol{B}\right|$\begin{tabular}{l|l}
$B \cos \chi_{b}$ \\
$B \sin \chi_{b}$ \\
0
\end{tabular}$=\boldsymbol{F} \mid \begin{aligned} & -J_{z 2} B \sin \chi_{b} \\
& J_{z 2} B \cos \chi_{b} \\
& -J_{y 2} B \cos \chi_{b}\end{aligned}$ 
and, after substituting the electric current components Eqs. (100a,b), they become:

$\left\{\begin{array}{l}F_{x}=-\left\{\left(\sigma_{P 2} N_{2} \sin \chi_{b}-f \sigma_{H 2} K_{2}\right) \varepsilon u_{1}-\eta \sigma_{P 2} N_{2} v_{1} \cos \chi_{b}\right\} B^{2} \sin \chi_{b} \\ F_{y}=\left\{\left(\sigma_{P 2} N_{2} \sin \chi_{b}-f \sigma_{H 2} K_{2}\right) \varepsilon u_{1}-\eta \sigma_{P 2} N_{2} v_{1} \cos \chi_{b}\right\} B^{2} \cos \chi_{b} \\ F_{z}=-\left\{\left(f \sigma_{P 2}-\sigma_{H 2} \sin \chi_{b}\right) \varepsilon u_{1}+\eta \sigma_{H 2} v_{1} \cos \chi_{b}\right\} K_{2} B^{2} .\end{array}\right.$

The magnitude of $\boldsymbol{F}$ is therefore such that:

$$
\begin{aligned}
\frac{F^{2}}{V_{1}^{2} B^{4}}= & \left\{\left(f \sigma_{H 2} K_{2}-\sigma_{P 2} N_{2} \sin \chi_{b}\right) \varepsilon \cos \chi_{1}+\eta \sigma_{P 2} N_{2} \sin \chi_{1} \cos \chi_{b}\right\}^{2} \\
& +\left\{\left(f \sigma_{P 2}-\sigma_{H 2} \sin \chi_{b}\right) \varepsilon \cos \chi_{1}+\eta \sigma_{H 2} \sin \chi_{1} \cos \chi_{b}\right\}^{2} K_{2}^{2} .
\end{aligned}
$$

In the limit of infinite $B$, the relations (26) become:

$$
\left\{\begin{array}{l}
\tilde{\sigma}_{P 2} \approx \frac{1}{s_{2}}, \\
\tilde{\sigma}_{H 2} \approx \frac{1}{s_{2} \beta_{i 2}}, \\
\tilde{\sigma}_{C 2} \approx \frac{1}{s_{2}}\left(1+\frac{1}{\beta_{i 2}^{2}}\right),
\end{array}\right.
$$

and therefore the constants $K_{2}$ and $N_{2}$ simplify as:

$$
\left\{\begin{array}{l}
K_{2} \approx \frac{\cos ^{2} \chi_{b}}{\cos ^{2} \chi_{b}\left(1+\frac{1}{s_{2}} \tan ^{2} \chi_{b}\right)} \approx 1-\frac{1}{s_{2}} \tan ^{2} \chi_{b} \\
N_{2} \approx \frac{\cos ^{2} \chi_{b}\left\{1+\frac{1}{s_{2}}\left(1+\frac{1}{\beta_{i 2}^{2}}\right) \tan ^{2} \chi_{b}\right\}}{\cos ^{2} \chi_{b}\left(1+\frac{1}{s_{2}} \tan ^{2} \chi_{b}\right)} \approx 1+\frac{1}{s_{2} \beta_{i 2}^{2}} \tan ^{2} \chi_{b}
\end{array}\right.
$$

The coefficient $f$ can be written:

$$
f \approx \frac{K_{2} \frac{S_{\rho \sigma}}{s_{2} \beta_{i 2}} \sin \chi_{b} \cos ^{2} \chi_{b}}{1+K_{2} \frac{S_{w \sigma}}{s_{2}} \cos ^{2} \chi_{b}} \approx \frac{\frac{S_{\rho \sigma}}{s_{2} \beta_{i 2}} \sin \chi_{b} \cos ^{2} \chi_{b}}{1+\frac{S_{w \sigma}}{s_{2}} \cos ^{2} \chi_{b}},
$$

where, from Eq. (105), we have assumed $K_{2} \approx 1, N_{2} \approx 1$. Now, the following estimates:

$\left\{\begin{array}{l}\frac{S_{\rho \sigma}}{s_{2}} \approx \frac{\sigma_{e 2} B^{2} \mathscr{L}_{\rho \sigma}}{m} \frac{m_{e} m_{i} v_{e} v_{i n}}{q_{e} q_{i} B^{2}} \approx \frac{m_{e} m_{i} v_{e} v_{i n} \sigma_{e 2} \mathscr{L}_{\rho \sigma}}{q_{e} q_{i} m} \\ \frac{S_{w \sigma}}{s_{2}} \approx \frac{\sigma_{e 2} B^{2} \mathscr{L}_{w \sigma}}{m} \frac{m_{e} m_{i} v_{e} v_{i n}}{q_{e} q_{i} B^{2}} \approx \frac{m_{e} m_{i} v_{e} v_{i n} \sigma_{e 2} \mathscr{L}_{w \sigma}}{q_{e} q_{i} m}\end{array}\right.$

show that these two ratios are independent of $B$. Since they are negligible compared to unity, we can approximate (106) as:

$f \approx \frac{S_{\rho \sigma}}{s_{2} \beta_{i 2}} \sin \chi_{b} \cos ^{2} \chi_{b}$.

Using these approximations, the magnitude of the Laplace force (103) can be written:

$$
\begin{aligned}
\frac{F^{2}}{\sigma_{e 2}^{2} V_{1}^{2} B^{4}} \approx & \frac{1}{s_{2}^{2}}\left\{\left(\frac{S_{\rho \sigma}}{s_{2} \beta_{i 2}^{2}} \cos ^{2} \chi_{b}-1\right) \varepsilon \sin \chi_{b} \cos \chi_{1}+\eta \sin \chi_{1} \cos \chi_{b}\right\}^{2} \\
& +\frac{1}{\left(s_{2} \beta_{i 2}\right)^{2}}\left\{\left(\frac{S_{\rho \sigma}}{s_{2}} \cos ^{2} \chi_{b}-1\right) \varepsilon \sin \chi_{b} \cos \chi_{1}+\eta \sin \chi_{1} \cos \chi_{b}\right\}^{2}
\end{aligned}
$$


Upon using the following equivalent expressions as $B \rightarrow+\infty$ :

$\left\{\begin{array}{l}\frac{1}{s_{2}^{2}} \propto B^{-4} \\ \frac{1}{\left(s_{2} \beta_{i 2}\right)^{2}} \propto B^{-6}\end{array}\right.$

we therefore conclude that only the first term is left in the relation (109):

$\frac{F^{2}}{\sigma_{e 2}^{2} V_{1}^{2} B^{4}} \approx \frac{1}{s_{2}^{2}}\left\{\left(\frac{S_{\rho \sigma}}{s_{2} \beta_{i 2}^{2}} \cos ^{2} \chi_{b}-1\right) \varepsilon \sin \chi_{b} \cos \chi_{1}+\eta \sin \chi_{1} \cos \chi_{b}\right\}^{2}$

The relations (107) show that the first term in parentheses is negligible compared to the second one, so that we finally obtain, after taking the square roots of both sides:

$F \approx \frac{\sigma_{e 2} V_{1} B^{2}}{s_{2}}\left|\mathcal{E} \sin \chi_{b} \cos \chi_{1}-\eta \sin \chi_{1} \cos \chi_{b}\right|$.

This is nothing else than the Laplace force without Hall effect, divided by the slip factor $s_{2}$ behind the shock.

\section{Appendix 5}

This appendix proposes a rigorous derivation of the inertial force $F_{l}$, which is used in the definition of the interaction parameter $S_{F}$, Eq. (66). A slightly different expression was used in our communication paper [4], and accordingly the interaction number $S_{F}$ was modified quantitatively, but not qualitatively. From the set $(3 b, c, d)$ and the conservation property $(10 a)$, we can estimate the total inertia force as:

$F_{I}^{2} \approx m^{2}\left\{\left(\frac{\mathrm{d} u}{\mathrm{~d} x}\right)^{2}+\left(\frac{\mathrm{d} v}{\mathrm{~d} x}\right)^{2}+\left(\frac{\mathrm{d} w}{\mathrm{~d} x}\right)^{2}\right\}$.

An order-of-magnitude estimate is obtained by writing the derivatives as finite differences across the shock:

$F_{I}^{2} \approx m^{2}\left\{\left(\frac{u_{2}-u_{1}}{\mathscr{L}_{\rho}}\right)^{2}+\left(\frac{v_{2}-v_{1}}{\mathscr{L}_{\rho}}\right)^{2}+\left(\frac{w_{2}}{\mathscr{L}_{\rho}}\right)^{2}\right\}$.

Then, using the jump relations $(27 a, b, c)$ and the characteristic scale $\mathscr{L}_{\rho}$ :

$F_{I}^{2} \approx \frac{m^{2}}{\mathscr{L}_{\rho}^{2}}\left\{(\varepsilon-1)^{2} u_{1}^{2}+(\eta-1)^{2} v_{1}^{2}+f \varepsilon u_{1}^{2}\right\}$,

and the projections (97), we obtain :

$F_{I}^{2} \approx \frac{m^{2} V_{1}^{2}}{\mathscr{L}_{\rho}^{2}}\left\{\left[(\varepsilon-1)^{2}+f \varepsilon\right] \cos ^{2} \chi_{1}+(\eta-1)^{2} \sin ^{2} \chi_{1}\right\}$.

Now, looking at Table 4, we see that $\varepsilon<<1, f<<1, \eta \approx 1$, so that Eq. (116) can be simplified into:

$F_{I} \approx \frac{m V_{1} \cos \chi_{1}}{\mathscr{L}_{\rho}}$,

and after substituting $m$, we finally obtain:

$F_{I} \approx \frac{\rho_{1} V_{1}^{2} \cos ^{2} \chi_{1}}{\mathscr{L}_{\rho}}$. 


\section{Appendix 6}

We develop hereafter the mathematical basis of a model of chemical non-equilibrium, in order to supplement the set of governing equations (10). For the 5-species model $\left(\mathrm{N}_{2}, \mathrm{O}_{2}, \mathrm{~N}, \mathrm{O}, \mathrm{NO}^{+}\right)$that we consider, the minimal set of reactions can be written (forward and backward):

$\left\{\begin{array}{l}\mathrm{N}_{2}+M_{1} \underset{\leftarrow}{\rightleftarrows} \mathrm{N}+M_{1} \\ \mathrm{O}_{2}+M_{2} \underset{\leftarrow}{\rightleftarrows} \mathrm{O}+M_{2} \\ \mathrm{~N}+\mathrm{O} \underset{\mathrm{NO}}{\leftarrow}+\mathrm{e}^{-}\end{array}\right.$

where the species $M_{1}$ and $M_{2}$ occurring in the first and second reactions may be $\mathrm{N}_{2}, \mathrm{O}_{2}, \mathrm{~N}$, or O.

Let us denote species concentrations by $C_{\mathrm{N} 2}, C_{M 1}, C_{\mathrm{O} 2}, C_{M 2}, C_{\mathrm{N}}, C_{\mathrm{O}}, C_{\mathrm{NO}+}$ and $N_{e}$ (electrons). Then, upon neglecting the diffusion terms, the conservation of species can be written in one dimension, for nitrogen, as [2][8]:

$\left\{\begin{array}{l}\frac{\mathrm{d}}{\mathrm{d} x}\left(u C_{\mathrm{N}_{2}}\right)=\left(k_{b 1} C_{\mathrm{N}}^{2}-k_{f 1} C_{\mathrm{N}_{2}}\right) C_{M_{1}} \\ \frac{\mathrm{d}}{\mathrm{d} x}\left(u C_{\mathrm{N}}\right)=2\left(k_{f 1} C_{\mathrm{N}_{2}}-k_{b 1} C_{\mathrm{N}}^{2}\right) C_{M_{1}}-k_{f 3} C_{\mathrm{N}} C_{\mathrm{O}}+k_{b 3} N_{e}^{2}\end{array}\right.$

for oxygen, as :

$\left\{\begin{array}{l}\frac{\mathrm{d}}{\mathrm{d} x}\left(u C_{\mathrm{O}_{2}}\right)=\left(k_{b 2} C_{\mathrm{O}}^{2}-k_{f 2} C_{\mathrm{O}_{2}}\right) C_{M_{2}} \\ \frac{\mathrm{d}}{\mathrm{d} x}\left(u C_{\mathrm{O}}\right)=2\left(k_{f 2} C_{\mathrm{O}_{2}}-k_{b 2} C_{\mathrm{O}}^{2}\right) C_{M_{2}}-k_{f 3} C_{\mathrm{N}} C_{\mathrm{O}}+k_{b 3} N_{e}^{2}\end{array}\right.$

and for charged species ( $\mathrm{NO}^{+}$and electrons), as :

$\left\{\begin{array}{l}\frac{\mathrm{d}}{\mathrm{d} x}\left(u C_{\mathrm{NO}^{+}}\right)=k_{f 3} C_{\mathrm{N}} C_{\mathrm{O}}-k_{b 3} C_{\mathrm{NO}^{+}} N_{e} \\ \frac{\mathrm{d}}{\mathrm{d} x}\left(u N_{e}\right)=k_{f 3} C_{\mathrm{N}} C_{\mathrm{O}}-k_{b 3} C_{\mathrm{NO}^{+}} N_{e}\end{array}\right.$

with usual notations for the forward and backward kinetic constants $k_{f}$ and $k_{b}$. At equilibrium $(u=0)$, we recover the usual relations satisfying Guldberg and Waage's law of mass action:

$\left\{\begin{array}{l}\frac{C_{\mathrm{N}}^{* 2}}{C_{\mathrm{N}_{2}}^{*}}=\frac{k_{f 1}}{k_{b 1}}=K_{1} \\ \frac{C_{\mathrm{O}}^{* 2}}{C_{\mathrm{O}_{2}}^{*}}=\frac{k_{f 2}}{k_{b 2}}=K_{2} \\ \frac{C_{\mathrm{N}^{+}}^{*} N_{e}^{*}}{C_{\mathrm{N}}^{*} C_{\mathrm{O}}^{*}}=\frac{k_{f 3}}{k_{b 3}}=K_{3}\end{array}\right.$

with the three equilibrium constants $K_{1}, K_{2}, K_{3}$.

In non-equilibrium $(u \neq 0)$, the linear combination $2 \times(120 a)+(120 b)+(122 a)$ yields:

$\frac{\mathrm{d}}{\mathrm{d} x}\left\{\left(2 C_{\mathrm{N}_{2}}+C_{\mathrm{N}}+C_{\mathrm{NO}^{+}}\right) u\right\}=0$,

the linear combination $2 \times(121 a)+(121 b)+(122 a)$ yields:

$\frac{\mathrm{d}}{\mathrm{d} x}\left\{\left(2 C_{\mathrm{O}_{2}}+C_{\mathrm{O}}+C_{\mathrm{NO}^{+}}\right) u\right\}=0$, 
and the difference $(122 a)-(122 b)$ yields:

$\frac{\mathrm{d}}{\mathrm{d} x}\left\{\left(C_{\mathrm{NO}^{+}}-N_{e}\right) u\right\}=0$.

Since there is no plasma ahead of the shock $\left(\left(C_{\mathrm{N}}\right)_{1}=0 ;\left(C_{\mathrm{O}}\right)_{1}=0 ;\left(C_{\mathrm{NO}}{ }^{+}\right)_{1}=0 ; N_{e 1}=0\right)$, the integration of Eqs. (124), (125), and (126) between $x_{1}$ (shock front) and $x$ (shock inside) leads to $(u \neq 0)$ :

$$
\left\{\begin{array}{l}
2 C_{\mathrm{N}_{2}}+C_{\mathrm{N}}+C_{\mathrm{NO}^{+}}=2 \frac{u_{1}}{u}\left(C_{\mathrm{N}_{2}}\right)_{1} \\
2 C_{\mathrm{O}_{2}}+C_{\mathrm{O}}+C_{\mathrm{NO}^{+}}=2 \frac{u_{1}}{u}\left(C_{\mathrm{O}_{2}}\right)_{1}
\end{array}\right.
$$

and :

$C_{\mathrm{NO}^{+}}=N_{\mathrm{e}}$.

Thus we recover the equation of conservation of nitrogen and oxygen atoms modified by the ratio $u_{1} / u$, Eqs. (127), and of electric charge, Eq. (128).

Now, following the same methodology as with velocity components and conductivities, we are led to extend relations (14) - (17) to the species concentrations, with the scale length $\mathscr{L}_{\mathbb{M}}$ characterizing molar mass changes through the shock:

$$
\left\{\begin{array} { l } 
{ C _ { \mathrm { N } _ { 2 } } = - \mathscr { L } _ { \mathcal { N } } \frac { \mathrm { d } C _ { \mathrm { N } _ { 2 } } } { \mathrm { d } x } } \\
{ C _ { \mathrm { O } _ { 2 } } = - \mathscr { L } _ { \mathcal { N } } \frac { \mathrm { d } C _ { \mathrm { O } _ { 2 } } } { \mathrm { d } x } }
\end{array} \left\{\begin{array} { l } 
{ C _ { \mathrm { N } } = \mathscr { L } _ { \mathcal { N } } \frac { \mathrm { d } C _ { \mathrm { N } } } { \mathrm { d } x } } \\
{ C _ { \mathrm { O } } = \mathscr { L } _ { \mathcal { N } } \frac { \mathrm { d } C _ { \mathrm { O } } } { \mathrm { d } x } }
\end{array} \left\{\begin{array}{l}
C_{\mathrm{NO}^{+}}=\mathscr{L}_{\mathcal{N}} \frac{\mathrm{d} C_{\mathrm{NO}^{+}}}{\mathrm{d} x} \\
N_{e}=\mathscr{L}_{\mathcal{N}} \frac{\mathrm{d} N_{\mathrm{e}}}{\mathrm{d} x}
\end{array}\right.\right.\right.
$$

These expressions involve minus signs for $\mathrm{N}_{2}$ and $\mathrm{O}_{2}$ species, because their concentrations $C_{\mathrm{N} 2}$ and $C_{\mathrm{O} 2}$ decrease across the shock, and plus signs for other species $\left(\mathrm{N}, \mathrm{O}, \mathrm{NO}^{+}\right)$and electrons, because their concentrations increase across the shock.

In a first step, we shall use these expressions in order to make a local analysis of the differential system (120), (121), and (122). Substituting relations (129) into Eqs. (120), (121), and (122) in order to remove the $x$ derivatives, we are led to define the compound length scales $\mathscr{L}_{\mu_{\rho}}$ et $\mathscr{L}_{\rho \mu_{0}}$ by the relations:

$\left\{\begin{array}{l}\frac{1}{\mathscr{L}_{N \rho}}=\frac{1}{\mathscr{L}_{N 6}}+\frac{1}{\mathscr{L}_{\rho}} \\ \frac{1}{\mathscr{L}_{\rho / 6}}=\frac{1}{\mathscr{L}_{\rho}}-\frac{1}{\mathscr{L}_{N}}\end{array}\right.$

Thus, inserting relation (129a) into Eq. (120a), solving for $C_{\mathrm{N} 2}$ and using the equilibrium constant (123a) yields:

$$
C_{\mathrm{N}_{2}}=\frac{C_{\mathrm{N}}^{2}}{K_{1}\left(1-\frac{u}{k_{f 1} C_{M_{1}} \mathscr{L}_{M \rho}}\right)} .
$$

Likewise, inserting relation (129b) into Eq. (121a), solving for $C_{\mathrm{O} 2}$ and using the equilibrium constant (123b) yields:

$$
C_{\mathrm{O}_{2}}=\frac{C_{\mathrm{O}}^{2}}{K_{2}\left(1-\frac{u}{k_{f 2} C_{M_{2}} \mathscr{L}_{\mu_{\rho} \rho}}\right)} .
$$

Applying the same procedure to Eq. (122b) and using the conservation of charge (128) yields:

$N_{e}=\frac{K_{3} C_{\mathrm{N}} C_{\mathrm{O}}}{N_{e}}-\frac{u}{k_{b 3} \mathscr{L}_{\rho \|_{6}}}$. 
Now, substituting (131), (132), and (133) into (127), using the conservation of charge (128) again, yields the system of two coupled quadratic equations for $C_{\mathrm{N}}$ and $C_{\mathrm{O}}$ :

$$
\left\{\begin{array}{l}
2 \frac{C_{\mathrm{N}}^{2}}{K_{1}\left(1-\frac{u}{k_{f 1} C_{M_{1}} \mathscr{L}_{M \rho} \rho}\right)}+\left(1+\frac{K_{3} C_{\mathrm{O}}}{N_{e}}\right) C_{\mathrm{N}}-2\left(\frac{u_{1}}{u}+\frac{u}{2 k_{b 3}\left(C_{\mathrm{N}_{2}}\right)_{1} \mathscr{L}_{\rho M}}\right)\left(C_{\mathrm{N}_{2}}\right)_{1}=0 \\
2 \frac{C_{\mathrm{O}}^{2}}{K_{2}\left(1-\frac{u}{k_{f 2} C_{M_{2}} \mathscr{L}_{M_{\rho} \rho}}\right)}+\left(1+\frac{K_{3} C_{\mathrm{N}}}{N_{e}}\right) C_{\mathrm{O}}-2\left(\frac{u_{1}}{u}+\frac{u}{2 k_{b 3}\left(C_{\mathrm{O}_{2}}\right)_{1} \mathscr{L}_{\rho \mu}}\right)\left(C_{\mathrm{O}_{2}}\right)_{1}=0
\end{array}\right.
$$

This set is complemented with the equation for $N_{\mathrm{e}}$ that can be also derived from (133):

$N_{e}^{2}-\frac{u}{k_{b 3} \mathscr{L}_{\rho \mathcal{M l}}} N_{e}-K_{3} C_{\mathrm{N}} C_{\mathrm{O}}=0$,

and whose physical solution can be written:

$N_{e}=\sqrt{K_{3} C_{\mathrm{N}} C_{\mathrm{O}}+\left(\frac{u}{2 k_{b 3} \mathscr{L}_{\rho M_{6}}}\right)^{2}}+\frac{u}{2 k_{b 3} \mathscr{L}_{\rho, l}}$.

Without advection $(u=0)$, resuming the calculations from the beginning results in the simple equation set:

$\left\{\begin{array}{l}2 \frac{C_{\mathrm{N}}^{* 2}}{K_{1}}+\left(1+\frac{K_{3} C_{\mathrm{O}}^{*}}{N_{e}^{*}}\right) C_{\mathrm{N}}^{*}-2\left(C_{\mathrm{N}_{2}}\right)_{1}=0 \\ 2 \frac{C_{\mathrm{O}}^{* 2}}{K_{2}}+\left(1+\frac{K_{3} C_{\mathrm{N}}^{*}}{N_{e}^{*}}\right) C_{\mathrm{O}}^{*}-2\left(C_{\mathrm{O}_{2}}\right)_{1}=0\end{array}\right.$

and:

$N_{e}^{*}=\sqrt{K_{3} C_{\mathrm{N}}^{*} C_{\mathrm{O}}^{*}}$.

This is the set of nonlinear equations that we solved for the description of the system in equilibrium, and used in the display of Fig. 15. The asterisks denote concentrations in the equilibrium state.

With advection $(u \neq 0)$, it is convenient to define four Damköhler numbers and a characteristic electron concentration $N_{e u}$, and recast Eqs. (134) and (136) as (75) and (76) in Sect. 4. As far as relation (31) holds, with $\zeta \approx 10$, the relations $(130)$ defining $\mathscr{L}_{\|_{0} \rho}$ and $\mathscr{L}_{\rho \mu_{0}}$ can be simplified as:

$\left\{\begin{array}{l}\mathscr{L}_{\mathbb{N}_{\rho}} \approx \mathscr{L}_{\rho} \\ \mathscr{L}_{\rho \mathbb{N}_{0}} \approx \mathscr{L}_{\rho}\end{array}\right.$ 


\section{References}

[1] Battesti, R., Beard, J., Böser, S., Bruyant, N., Budker, D., Crooker, S.A., Dawg, E. J., Flambaum, V.V., Inada, T., Irastorza, I.G., Karbstein, F., Kim, D.L., Kozlov, M.G., Melhem, Z., Phipps, A., Pugnat, P., Rikken, G., Rizzo, C., Schott, M.,Semertzidis, Y.K., ten Kate, H.H.J., Zavattini, G.: High magnetic fields for fundamental physics. Phys. Rep. 765-766, 1-39 (2018)

[2] Benson, S.W.: The foundations of chemical kinetics. McGraw-Hill (1960)

[3] Berton, R.P.H.: Analytic model of a resistive magnetohydrodynamic shock without Hall effect. J. Fluid Mech. 842, 273-322 (2018)

[4] Berton, R.P.H.: Analytic model of a resistive MHD shock. In : HiSST 2018, Moscow, 26-29 November 2018, Paper \# 2999 (2018)

[5] Bityurin, V.A., Bocharov, A.N.: Magnetohydrodynamic interaction in hypersonic air flow past a blunt body. Fluid Dyn. 41 (5), 843-856 (2006)

[6] Bityurin, V.A., Bocharov, A.N.: MHD heat flux mitigation in hypersonic flow around a blunt body with ablating surface. J. Phys. D 51, 264001 (2018)

[7] Braiding, C.R., Wardle, M.: The Hall effect in star formation. Mon. Not. R. Astron. Soc. 422, 261-281 (2012)

[8] Candler, G.V.: On the computation of shock shapes in nonequilibrium hypersonic flows. In : 27th Aerospace Sciences Meeting, 9-12 January 1989, Reno (Nevada), AIAA Paper 1989-312 (1989)

[9] Candler, G.V., MacCormack, R.W.: Computation of weakly ionized hypersonic flows in thermochemical nonequilibrium. J. Thermophys. Heat Trans. 5(3), 266-273 (1991)

[10] Cavus, H.: On the effects of viscosity on the shock waves for a hydrodynamical case - Part I: basic mechanism. Adv. Astron. 2013, 582965, 1-6 (2013)

[11] Cowling, T.G.: The electrical conductivity of an ionized gas in a magnetic field, with applications to the solar atmosphere and the ionosphere. Proc. Roy. Soc. A 183 (995), 453-479 (1945)

[12] Cristofolini, A., Borghi, C.A., Schettino, A., Battista, F.: Numerical rebuilding of MHD tests in an unseeded Mach 10 air flow around a blunt body. In : 43rd AIAA Plasmadynamics and Lasers Conference, 25-28 June 2012, New Orleans (Louisiana), AIAA Paper 2012-2733 (2012)

[13] D'Angola, A., Colonna, G., Gorse, C., Capitelli, M.: Thermodynamic and transport properties in equilibrium air plasmas in a wide pressure and temperature range. Eur. Phys. J. D 46, 129-150 (2008)

[14] D'Angola, A., Colonna, G., Gorse, C., Capitelli, M.: Thermodynamic properties of high temperature air in local thermodynamic equilibrium: II Accurate analytical expression for electron molar fractions. Eur. Phys. J. D 65, 453-457 (2011)

[15] Dorelli, J.C.: Effects of Hall electric fields on the saturation of forced antiparallel magnetic field merging. Phys. Plasmas 10, 3309-3314 (2003).

[16] Ericson, W.B., Maciulaitis, A.: Investigation of magnetohydrodynamic flight control. J. Spacecr. 1 (3), $283-$ 289 (1964)

[17] Farbar, E., Boyd, I.D., Martin, A.: Numerical prediction of hypersonic flowfields including effects of electron translational nonequilibrium. J. Thermophys. Heat Trans. 27(4), 593-606 (2013)

[18] Fomichev, V., Yadrenkin, M.: Strong MHD-interaction in hypersonic flows near bodies. AIP Conf. Proc., 1893, 030020 (2017)

[19] Fujino, T., Sakakihara, R., Ishikawa, M.: Numerical simulation of magnetohydrodynamic heat shield in reentry flight with considering induced magnetic field. J.Sp.Tech.Sci. 25 (2), 55-69 (2011)

[20] Fujino, T., Ishikawa, M.: Feasibility of an onboard surface Hall magnetohydrodynamic power generator in reentry flight. J. Prop. Power 25 (1), 83-93 (2009)

[21] Fujino, T., Ishikawa, M.: Performance characteristics of onboard Hall-type magnetohydrodynamic generator during Earth reentry flight. J. Prop. Power 31 (2), 737-749 (2015)

[22] Galtier, S.: Introduction to modern magnetohydrodynamics. Cambridge University Press (2016)

[23] Grier, N.T., Sands, N.: Regime of frozen boundary layers in stagnation region of blunt reentry bodies. NASA TN-D 865 (1961) 
[24] Gupta R.N., Yoss J.M., Thompson R.A., Lee K.P.: A review of reaction rates and thermodynamic and transport properties for an 11-species air model for chemical and thermal nonequilibrium calculations to 30000 K. NASA RP-1232 (1990)

[25] Hasimoto, H.: Swirl of a conducting gas due to the Hall effect. J. Phys. Soc. Jpn 19(8), 1457-1463 (1964)

[26] Hau, L.-N., Sonnerup, B.U.Ö.: On the structure of resistive MHD intermediate shocks. J. Geophys. Res., 94 (A6), 6539-6551 (1989)

[27] Howe, G. G.: Limitations of Hall MHD as a model for turbulence in weakly collisional plasmas. Nonlinear Proc. Geophys. 16, 219-232 (2009)

[28] Hugoniot, H.: Mémoire sur la propagation du mouvement dans les corps et spécialement dans les gaz parfaits. Deuxième partie. $58^{\text {ème }}$ cahier, 1-125 (1889)

[29] Kandus, A., Vasconcelos, M.J., Cerqueira, A.H.: On the mean field dynamo with Hall effect. Astron. Astrophys. 452, 773-779 (2006)

[30] Keidar, M., Kim, M., Boyd, I.: Electromagnetic reduction of plasma density during atmospheric reentry and hypersonic flights. J. Sp. Rock. 45 (3), 445-453 (2008)

[31] Lepschy, A., Viaro, U.: Complexity and feedback. In : Isidori, A., Tarn, T.J. (eds.) Systems, models and feedback: theory and applications. Proceedings of a U.S.-Italy Workshop in honor of Professor Antonio Ruberti, Capri, 15-17 June 1992, Progress in Systems and Control Theory, Vol. 12, Springer Science+Business Media, LLC, pp. 111-126 (1992)

[32] Macheret, S.O., Shneider, M.N., Candler, G.V.: Modeling of MHD power generation on board reentry vehicles. In : 42nd AIAA Aerospace Sciences Meeting and Exhibit, 5-8 January 2004, Reno (Nevada), AIAA Paper 2004-1024 (2004)

[33] Moretti, G.: Thirty-six years of shock fitting. Comput. Fluids 31 (4), 719-723 (2002)

[34] Onofri, M., Paciorri, R. (eds.) : Shock fitting. Classical techniques, recent developments, and memoirs of Gino Moretti. Springer Nature, Cham (2017)

[35] Otsu, H., Matsushita, K., Konigorski, D., Funaki, I., Abe, T.: Reentry heating mitigation by utilizing the Hall effect. In : 35th AIAA Plasmadynamics and Lasers Conference, 28 June-1 July 2004, Portland (Oregon), AIAA Paper 2004-2167 (2004)

[36] Otsu, H., Konigorski, D., Abe, T.: Influence of Hall effect on electrodynamic heat shield system for reentry vehicles. AIAA J. 48 (10), 2177-2186 (2010)

[37] Park, C.: Assessment of two-temperature kinetic model for ionizing air. J. Thermophys., 3(3), 233-244 (1989)

[38] Pepe, R., Bonfiglioli, A., D’Angola, A., Colonna, G., Paciorri, R.: Shock-fitting versus shock-capturing modeling of strong shocks in nonequilibrium plasmas. IEEE Trans. Plasma Sci. 42 (10), 2526-2527 (2014)

[39] Poggie, J., Gaitonde, D.V.: Magnetic control of flow past a blunt body: numerical validation and exploration. Phys. Fluids 14 (5), 1720-1731 (2002)

[40] Porter, R.W., Cambel, A.B.: Hall effect in flight magnetogasdynamics. AIAA J. 5 (12), 2208-2213 (1967)

[41] Poumirol, J.M., Escoffier, W., Kumar, A., Goiran, M., Raquet, B., Broto, J.M.: Electron-hole coexistence in disordered graphene probed by high-field magneto-transport. New J. Phys. 12, 083006 (2010)

[42] Rankine, W.J.M.: On the thermodynamic theory of waves of finite longitudinal disturbance. Proc. R. Soc. Lond. 160, 277-288 (1870)

[43] Salas, M.D.: A shock-fitting primer. CRC Press, Boca Raton (2010)

[44] Satheesh, K., Jagadeesh, G., Reddy, K.P.J.: High speed Schlieren facility for visualization of flow fields in hypersonic shock tunnels. Curr. Sci., 92(1), 56-60 (2007)

[45] Srinivasan, S., Tannehill, J.C., Weilmuenster, K.J.: Simplified curve fits for the thermodynamic properties of equilibrium air. NASA RP 1181 (1987)

[46] Sutton, G.W., Sherman, A.: Engineering Magnetohydrodynamics. Dover, Mineola (2006) 
Figures

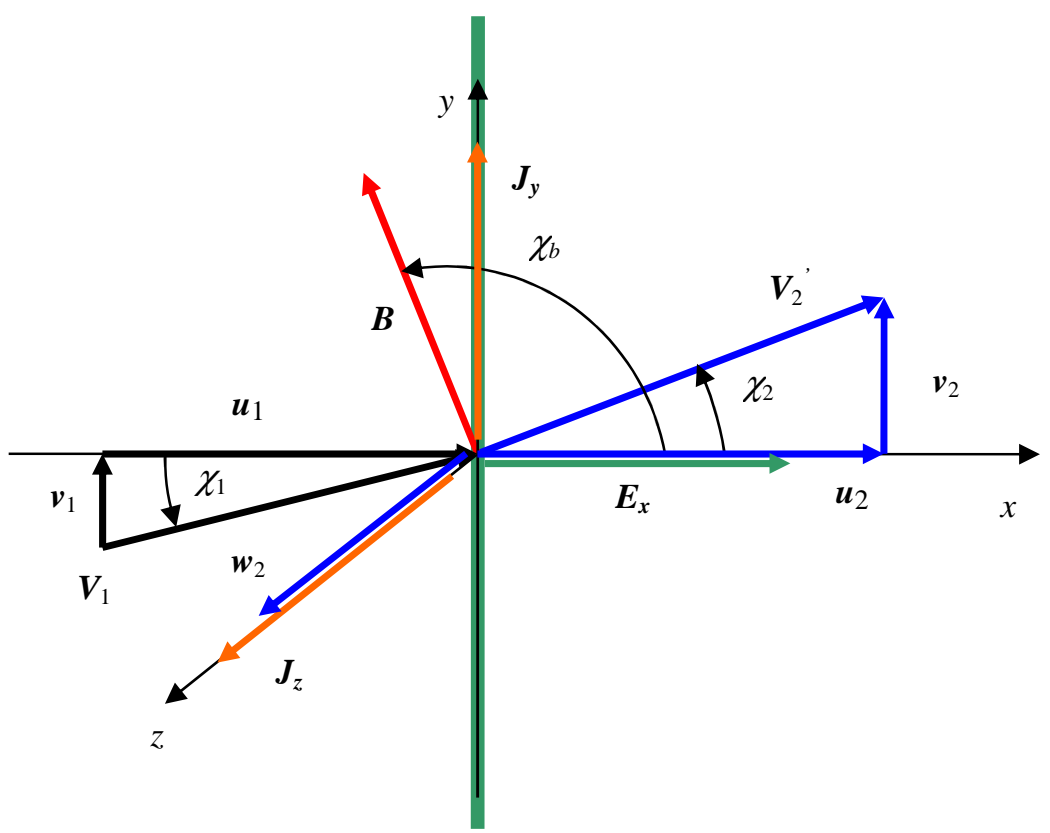

Shock plane

Fig. 1 Plane oblique shock 


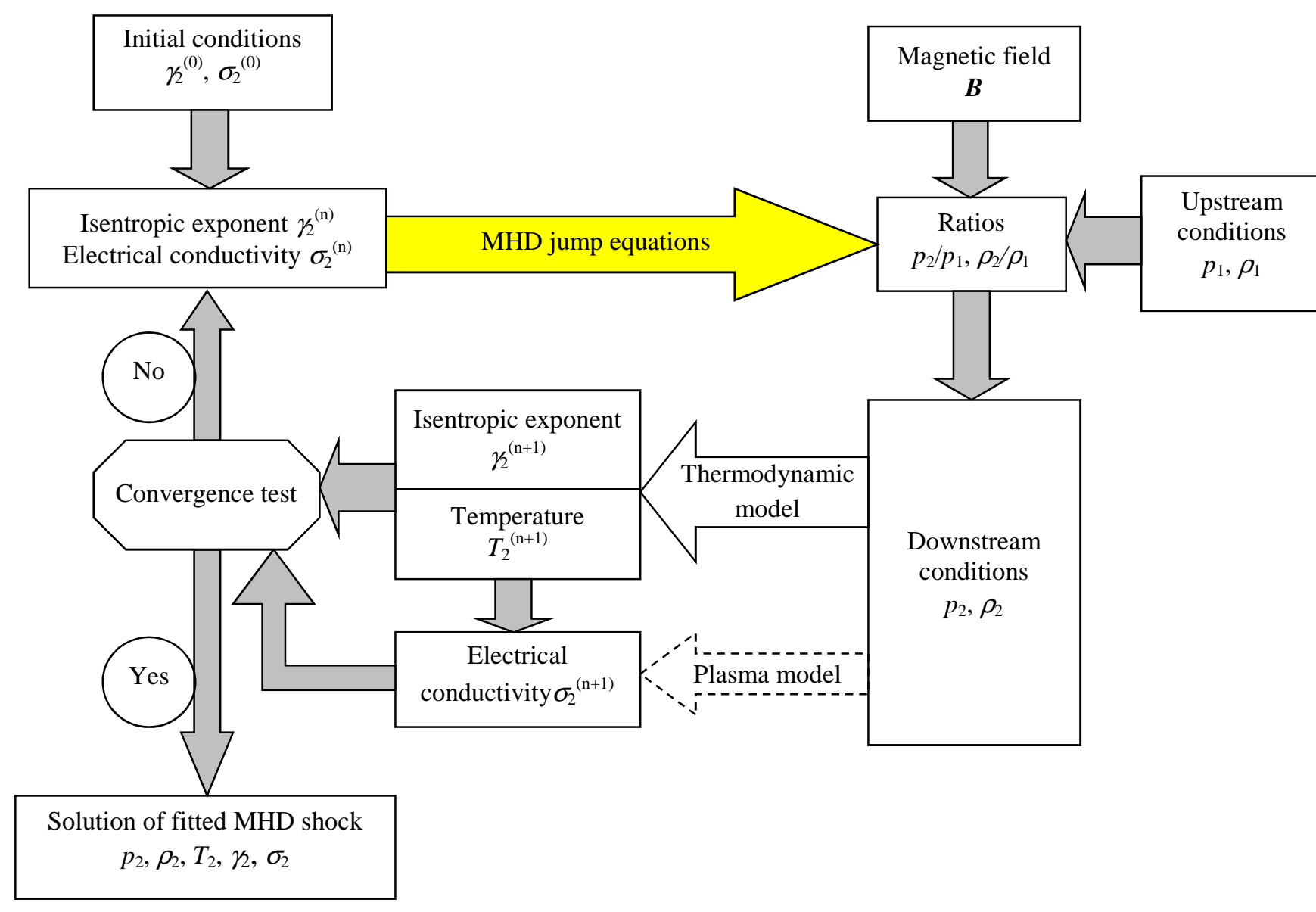

Fig. 2 Flow chart of MHD plane shock fitting with Hall effect. 


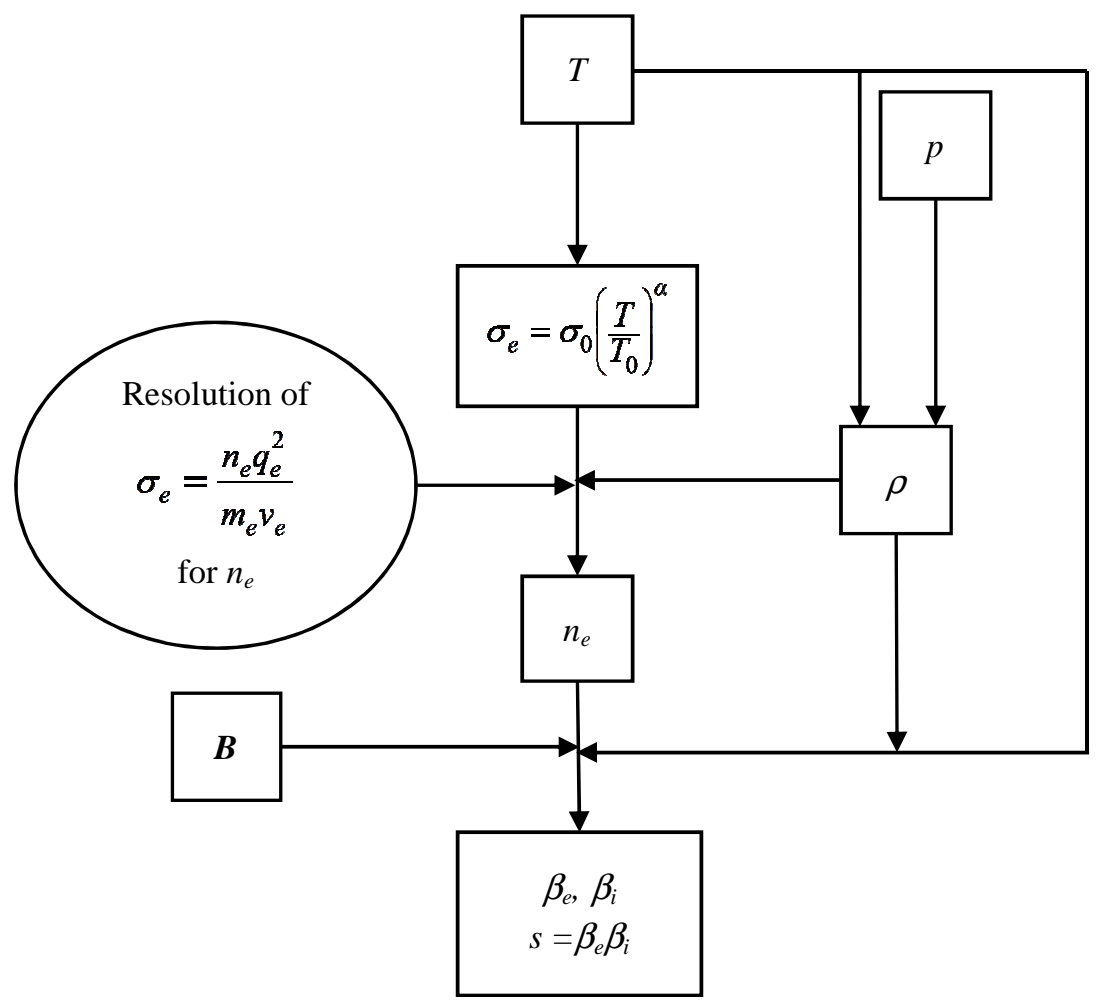

Fig. 3 Calculation of the Hall parameters $\beta_{e}, \beta_{i}$, and slip factor $s$ from the ordinary electrical conductivity $\sigma_{e}$ and ambient physical conditions. 


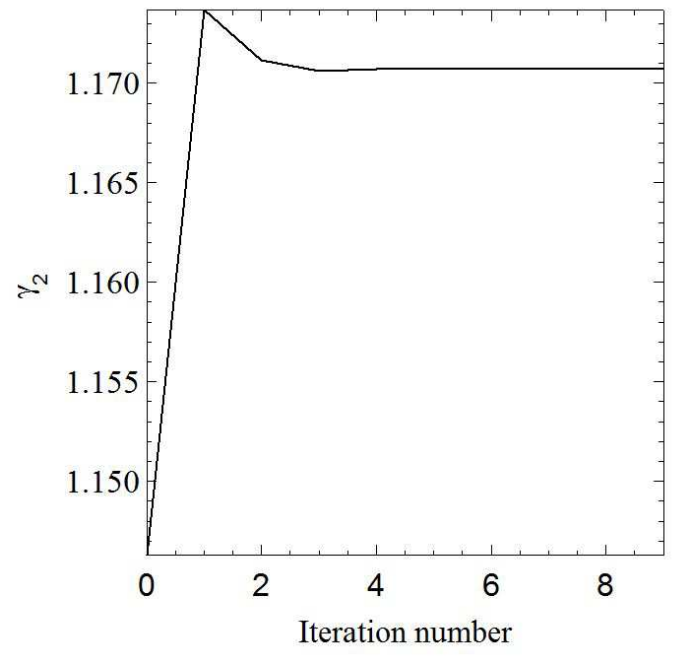

(a)

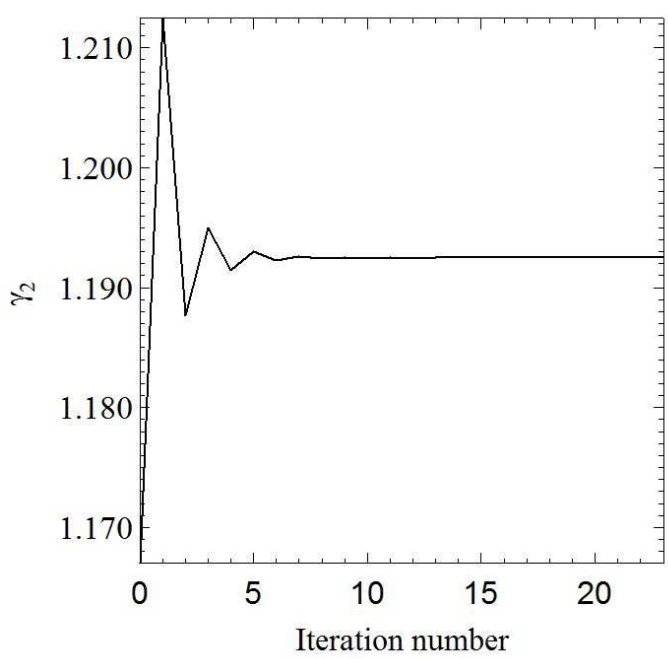

(c)

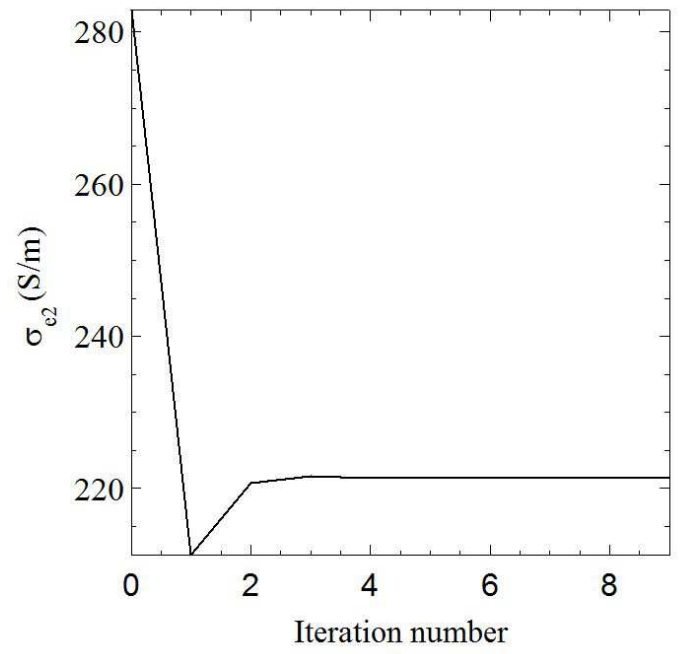

(b)

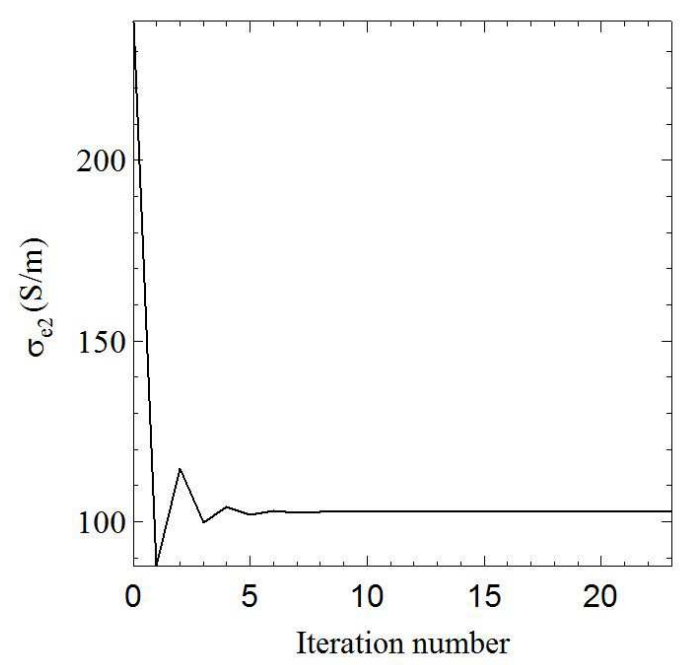

(d)

Fig. 4 Convergence plots of isentropic exponent $\gamma_{2}(\boldsymbol{a}, \boldsymbol{c})$ and ordinary electrical conductivity $\sigma_{e 2}(\boldsymbol{b}, \boldsymbol{d})$ in shock fitting, without $(\boldsymbol{a}, \boldsymbol{b})$ and with $(\boldsymbol{c}, \boldsymbol{d})$ Hall effect, using the procedures of Figs. 2 and 3 for a resistive MHD plane oblique shock $\left(\chi_{1}=60^{\circ} ; \chi_{b}=150^{\circ} ; B=1 \mathrm{~T} ; \alpha=2 ; \zeta=10\right)$ with physical conditions at $65 \mathrm{~km}$ and Mach 23.

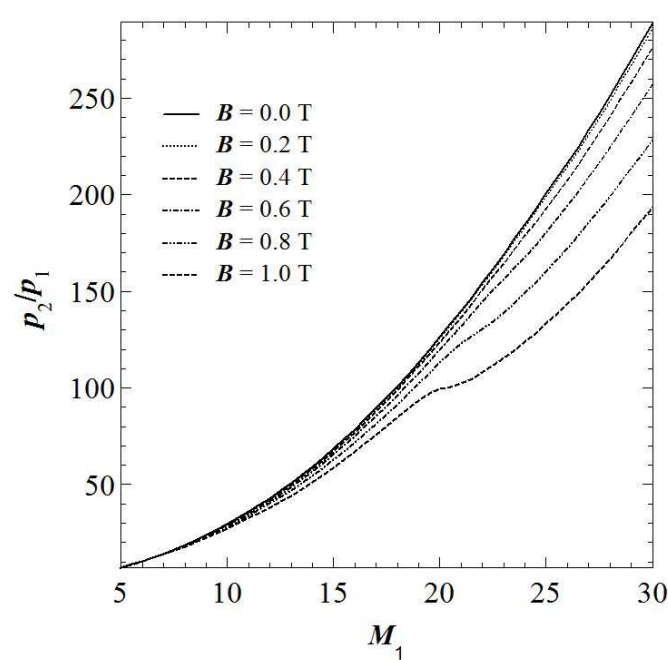

(a)

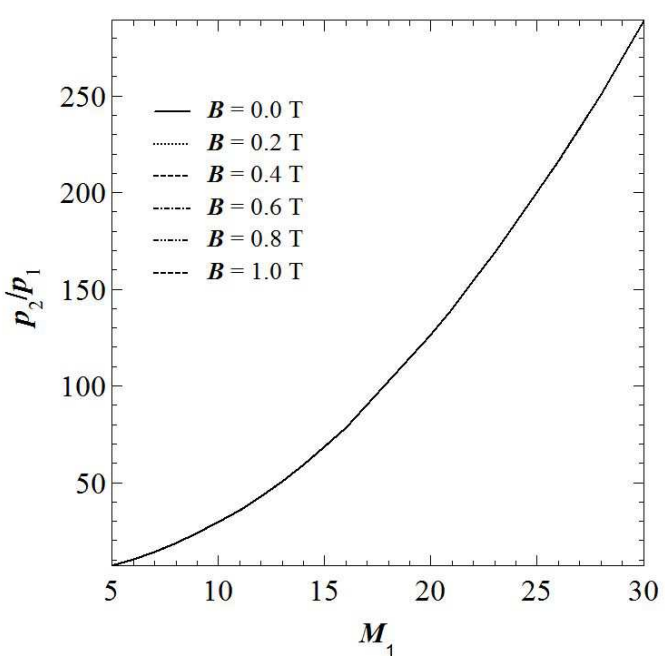

(b)

Fig. 5 Variation of $p_{2} / p_{1}$ as a function of $M_{1}$ and $B$ : without $(\boldsymbol{a})$ and with $(\boldsymbol{b})$ Hall effect. 


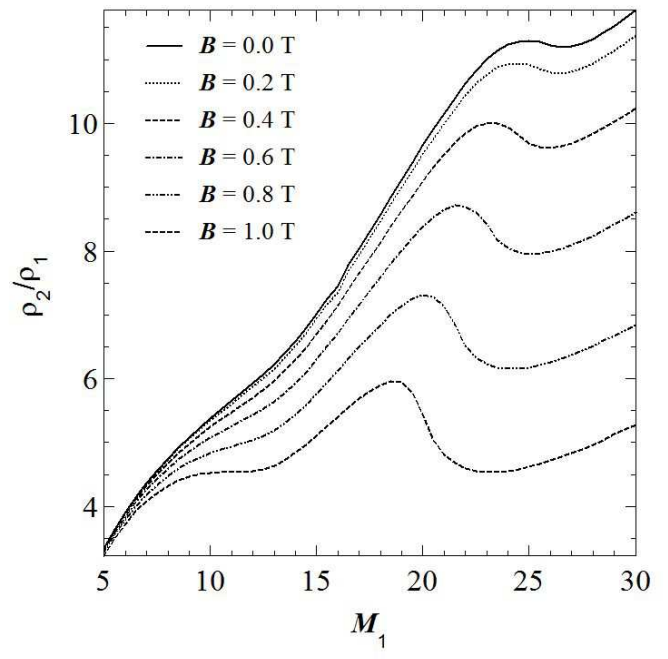

(a)

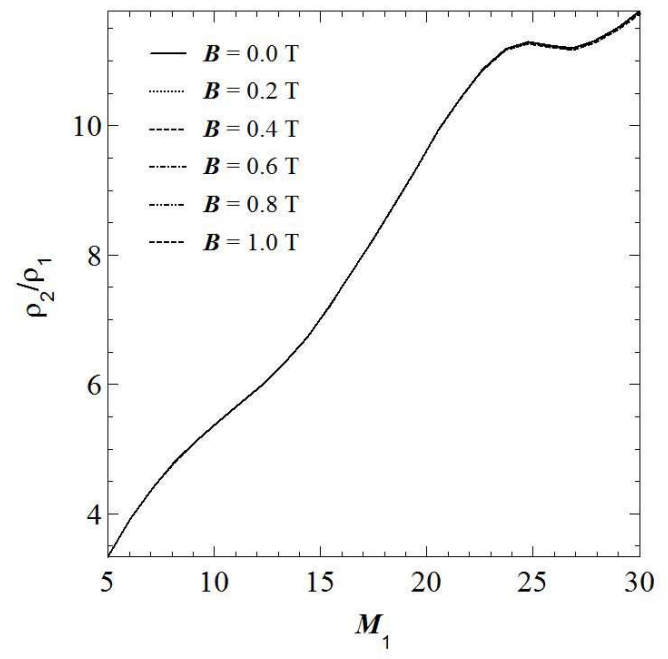

(b)

Fig. 6 Variation of $\rho_{2} / \rho_{1}$ as a function of $M_{1}$ and $B$ : without $(\boldsymbol{a})$ and with $(\boldsymbol{b})$ Hall effect.

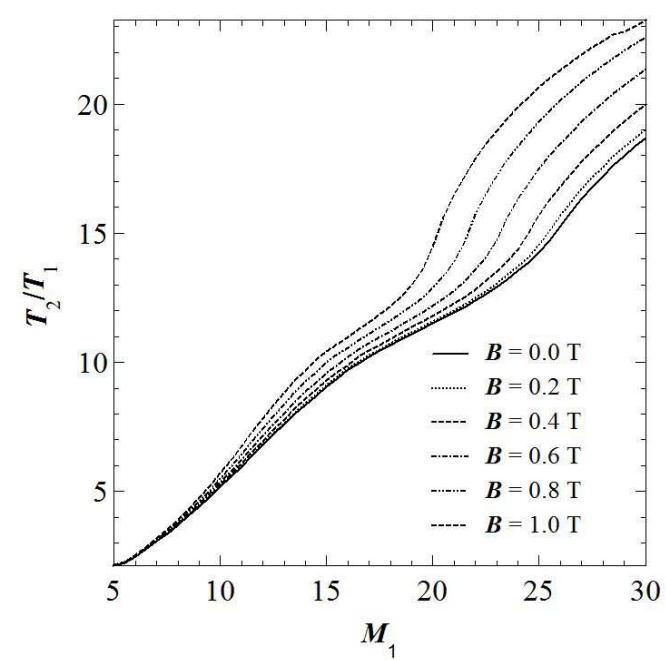

(a)

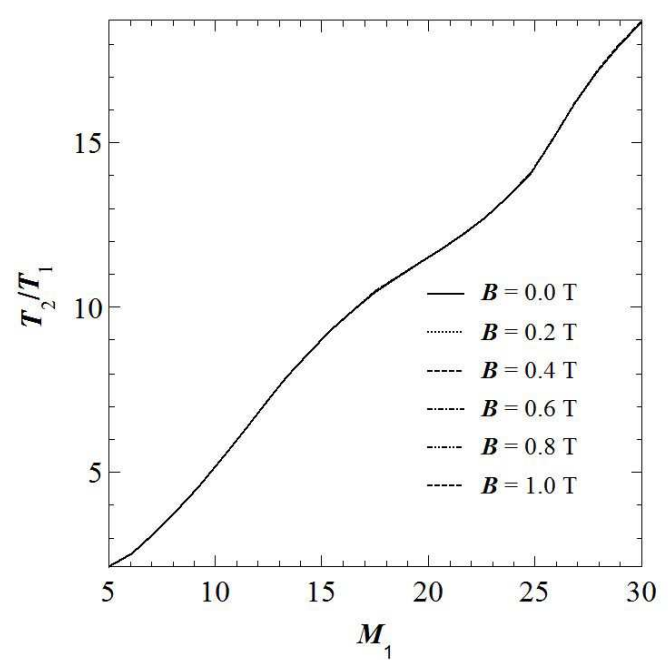

(b)

Fig. 7 Variation of $T_{2} / T_{1}$ as a function of $M_{1}$ and $B$ : without $(\boldsymbol{a})$ and with $(\boldsymbol{b})$ Hall effect.

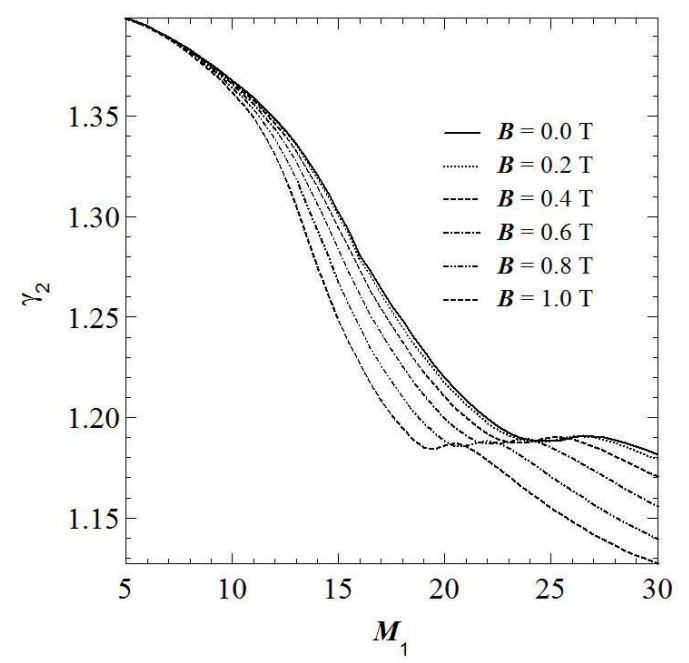

(a)

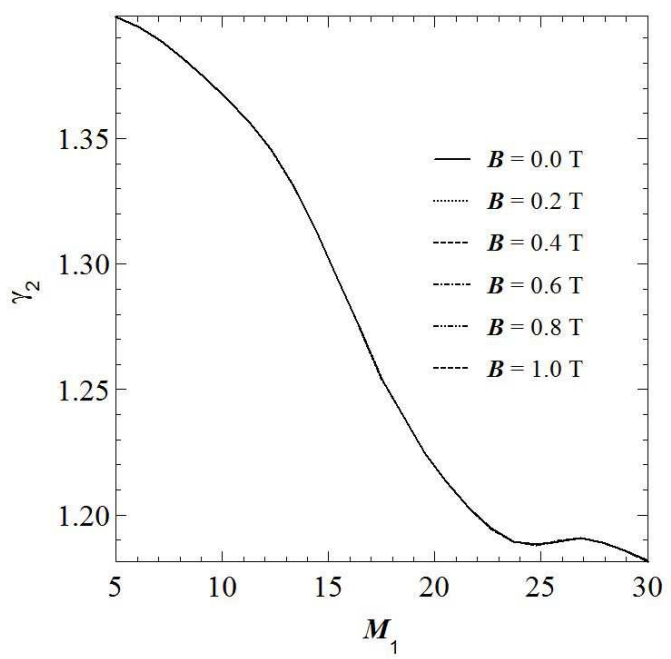

(b)

Fig. 8 Variation of $\gamma_{2}$ as a function of $M_{1}$ and $B$ : without $(\boldsymbol{a})$ and with $(\boldsymbol{b})$ Hall effect. 


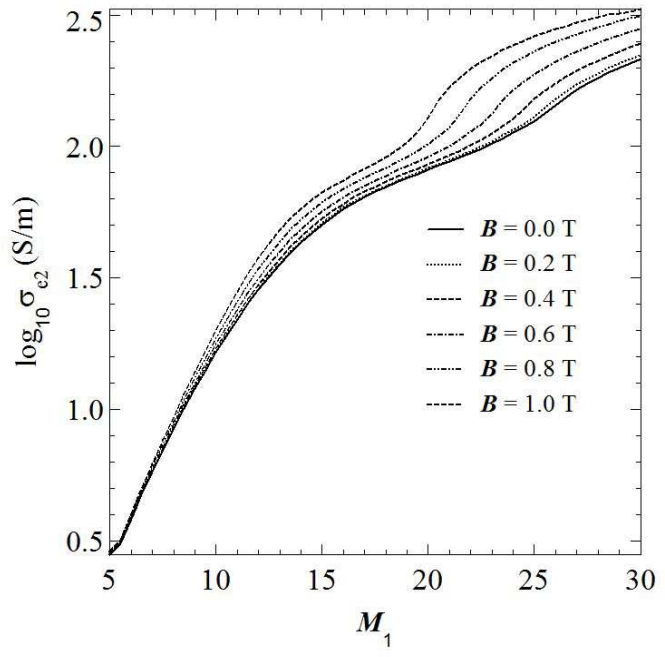

(a)

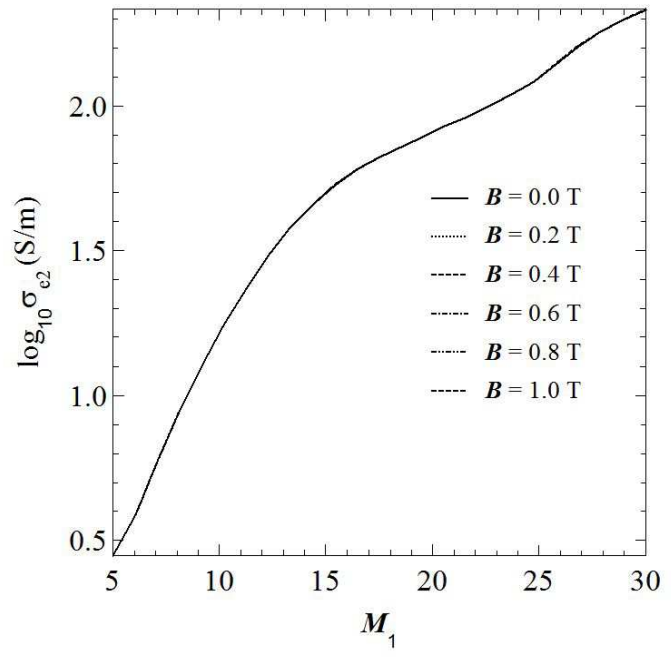

(b)

Fig. 9 Variation of $\sigma_{e 2}$ as a function of $M_{1}$ and $B$ : without $(\boldsymbol{a})$ and with $(\boldsymbol{b})$ Hall effect.

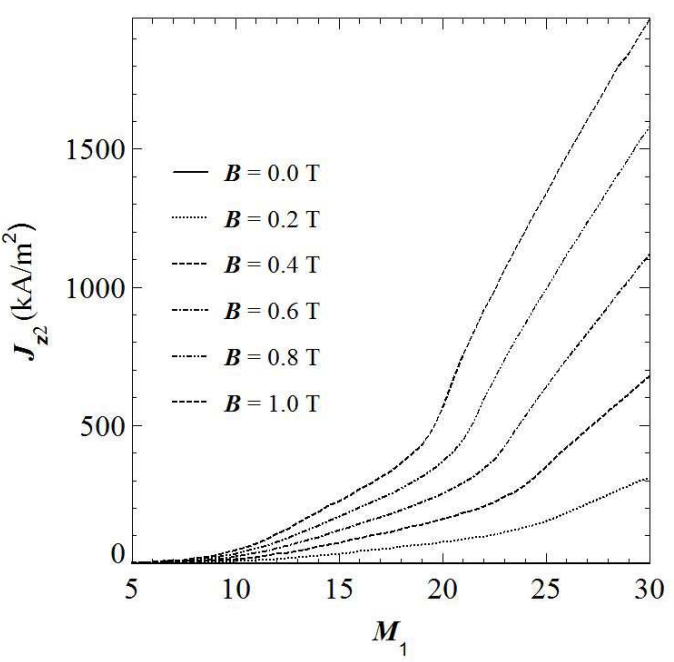

(a)

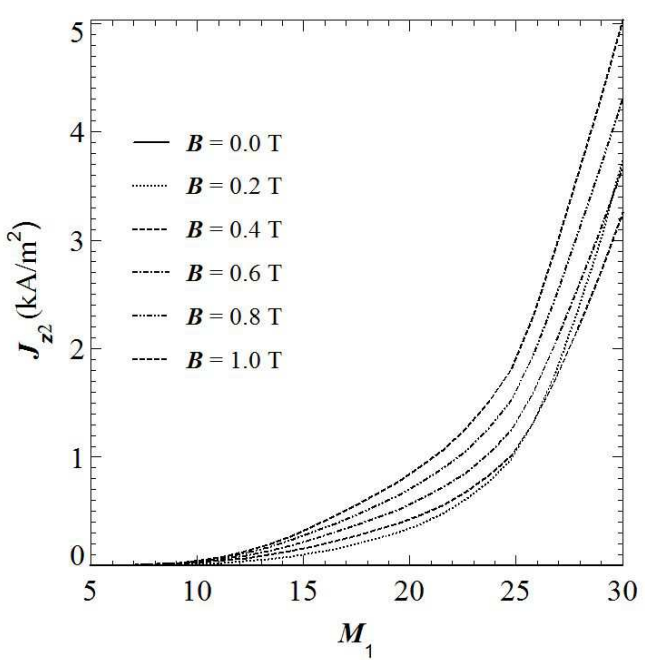

(b)

Fig. 10 Variation of $J_{z 2}$ as a function of $M_{1}$ and $B$ : without (a) and with (b) Hall effect.

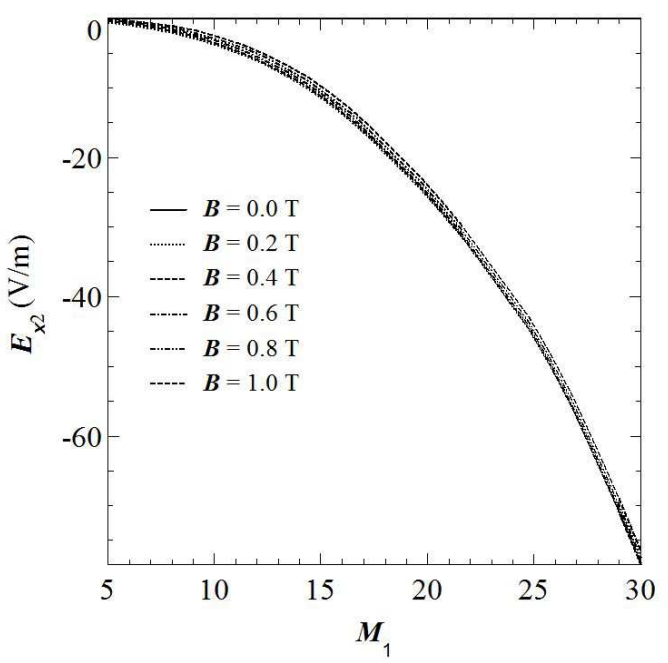

(a)

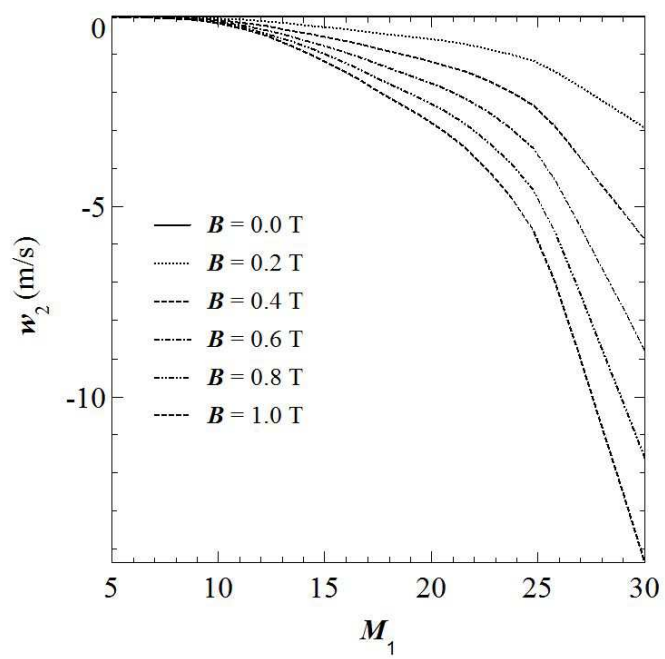

(b)

Fig. 11 Variations of $E_{x 2}(\boldsymbol{a})$ and $w_{2}(\boldsymbol{b})$ as functions of $M_{1}$ and $B$, with Hall effect. 


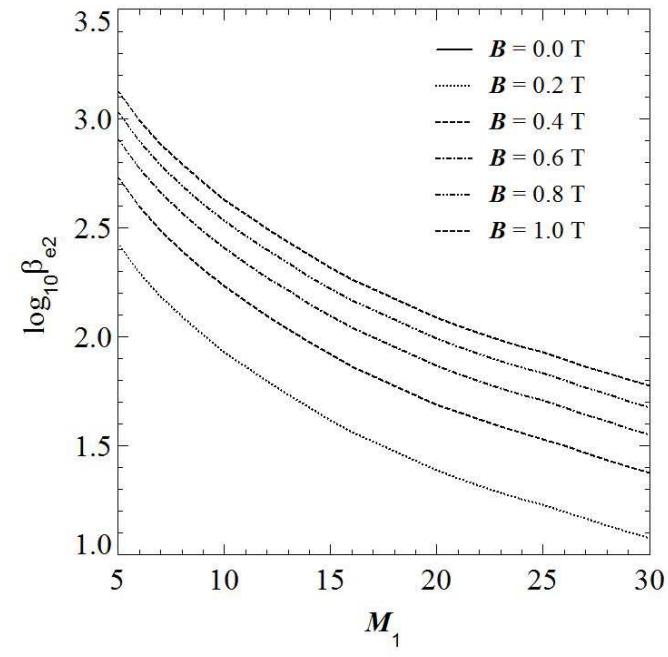

(a)

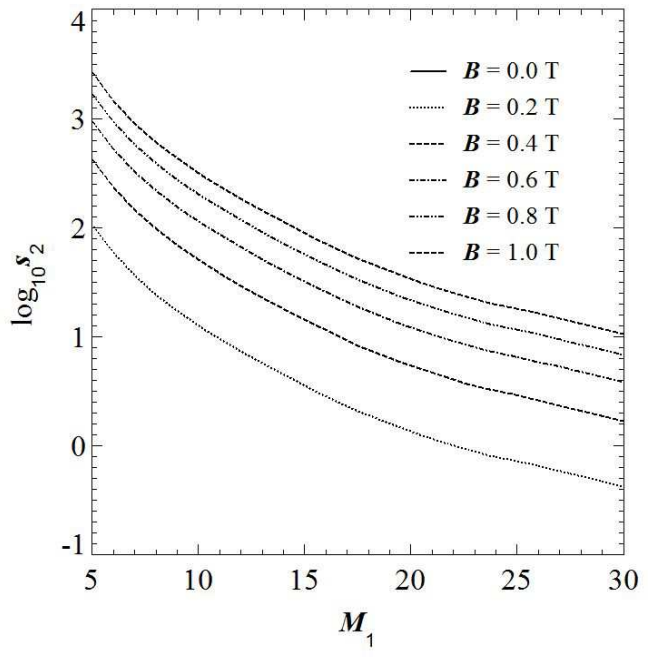

(b)

Fig. 12 Variations of $\beta_{e 2}(\boldsymbol{a})$ and $s_{2}(\boldsymbol{b})$ as functions of $M_{1}$ and $B$, with Hall effect.

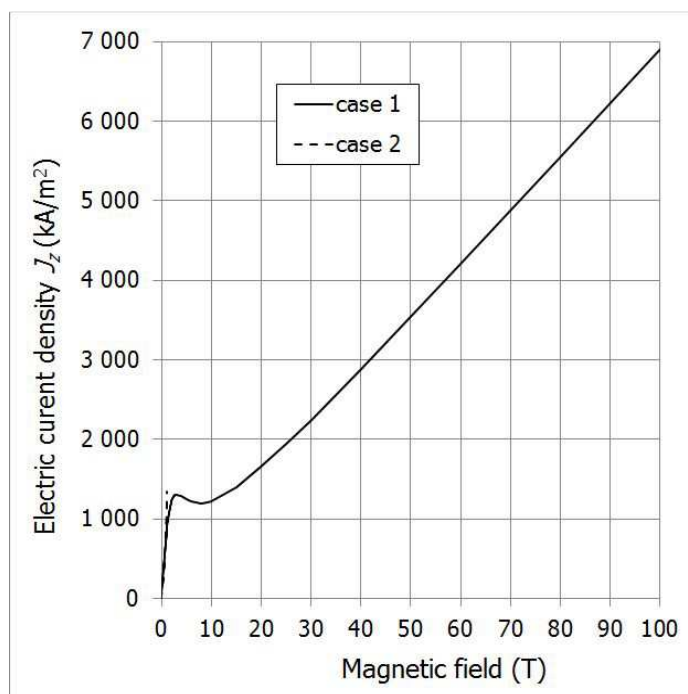

(a)

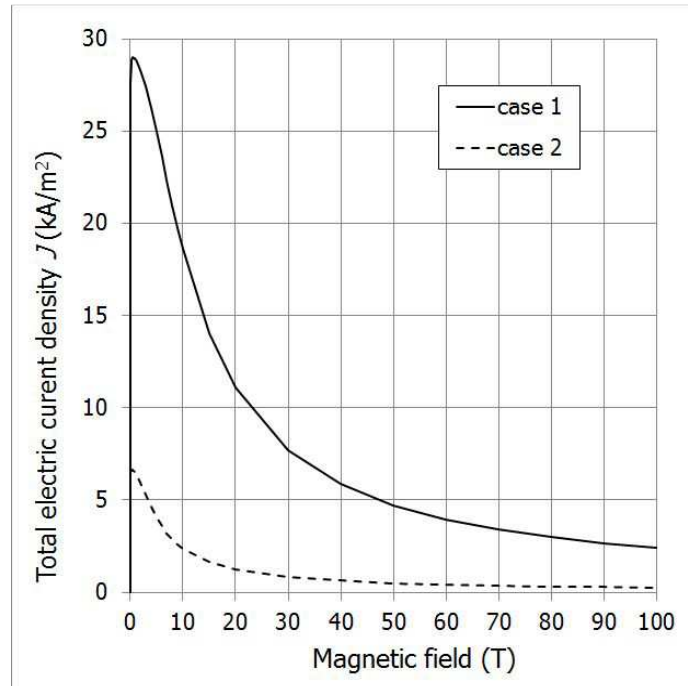

(b)

Fig. 13 Variation the total electric current density as a function of the magnetic field strength $B$ for the two geometrical configurations considered in $\S 3.2$ : without $(\boldsymbol{a})$ and with $(\boldsymbol{b})$ Hall effect $\left(M_{1}=23\right)$. 


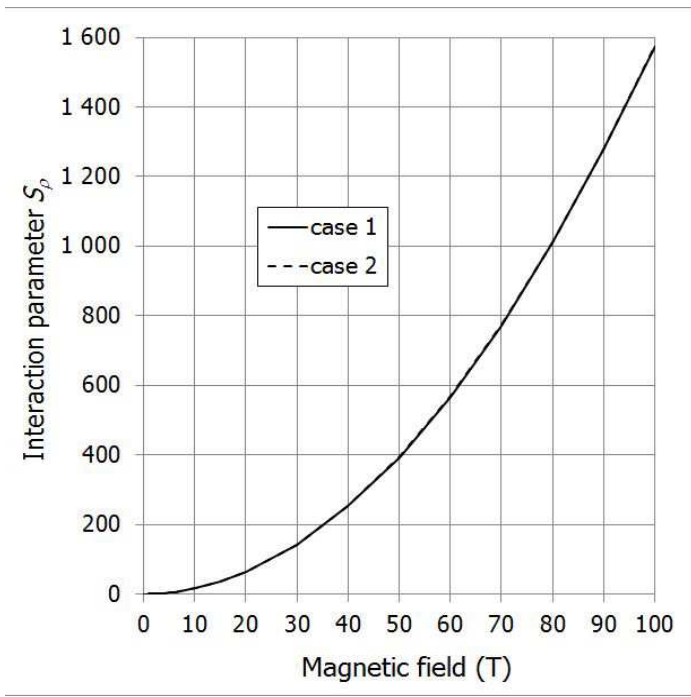

(a)

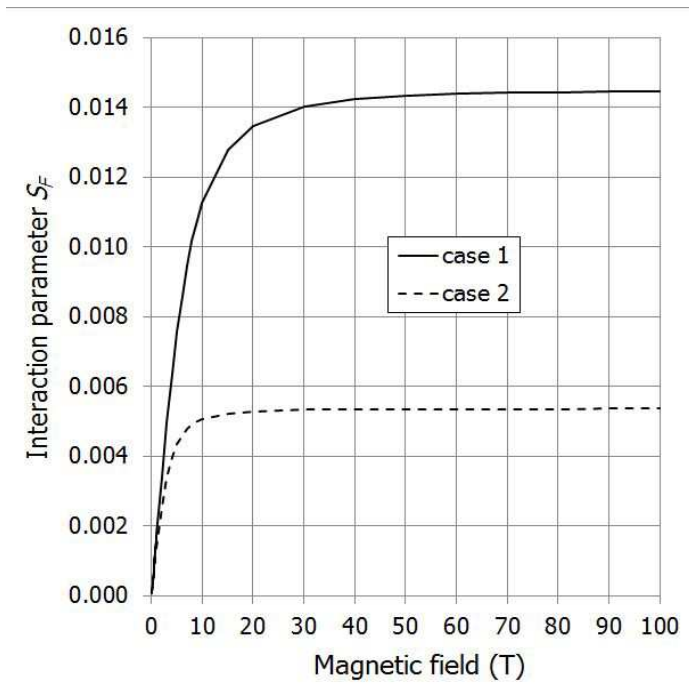

(b)

Fig. 14 Variations of the usual interaction parameter $S_{\rho}(\boldsymbol{a})$ and of the Laplace-force based one $S_{F}(b)$ as functions of the magnetic field strength $B$ for the two cases considered in presence of Hall effect $\left(M_{1}=23\right)$.

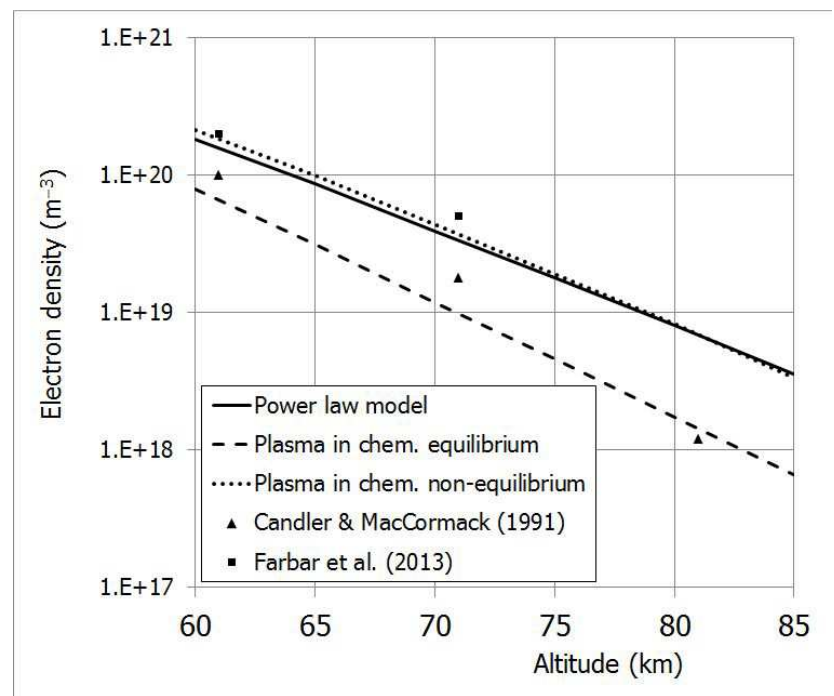

Fig. 15 Variations of the downstream electron density $n_{e}$ as a function of altitude for a resistive MHD normal shock $\left(\chi_{1}=0^{\circ} ; \chi_{b}=180^{\circ} ; B=1 \mathrm{~T} ; \alpha=2 ; \zeta=10\right)$ and a power law conductivity. The altitude sampling step is $5 \mathrm{~km}$. Also plotted are results from Candler \& McCormack (1991) and Farbar et al. (2013), and values $n_{e}{ }^{*}$ and $n_{e}{ }^{* *}$ obtained with a 5-species plasma model in chemical equilibrium and non-equilibrium respectively. 
Table 1 Nomenclature of symbols and notations

\begin{tabular}{|c|c|}
\hline Symbol & Meaning \\
\hline$a, b, c, d, f$ & Coefficients of MHD velocity jump \\
\hline $\boldsymbol{B}, B$ & Magnetic induction (field) vector and strength $(\mathrm{T})$ \\
\hline$c_{s}$ & Sound speed $\left(\mathrm{m} \mathrm{s}^{-1}\right)$ \\
\hline $\boldsymbol{E}$ & Electric field $\left(\mathrm{V} \mathrm{m}^{-1}\right)$ \\
\hline$f_{e}$ & Collision frequency of electrons $(\mathrm{Hz})$ \\
\hline$J, J$ & Electric current vector and magnitude $\left(\mathrm{A} \mathrm{m}^{-2}\right)$ \\
\hline $\mathscr{L}$ & Characteristic length $(\mathrm{m})$; shock thickness \\
\hline $\begin{array}{l}k_{f}, k_{b} \\
\ell\end{array}$ & $\begin{array}{l}\text { Forward and backward kinetic rate constants }\left(\mathrm{m}^{3} \mathrm{~mol}^{-1} \mathrm{~s}^{-1}\right) \\
\text { Mean free molecular path }(\mathrm{m})\end{array}$ \\
\hline$M$ & Mach number $\left(=V / c_{s}\right)$ \\
\hline$m$ & Normal mass flow rate $\left(=\rho_{1} u_{1}=\rho_{2} u_{2}\right)$ \\
\hline$m_{e}$ & Electron mass $\left(=0.91091 \times 10^{-30} \mathrm{~kg}\right)$ \\
\hline$N_{e}, n_{e}$ & Electron concentration $\left(\mathrm{mol} \mathrm{m}{ }^{-3}\right)$ and density $\left(\mathrm{m}^{-3}\right)$ respectively \\
\hline$p$ & Thermal pressure $(\mathrm{Pa})$ \\
\hline$q_{e}$ & Electron charge $\left(=1.60210 \times 10^{-19} \mathrm{C}\right)$ \\
\hline$R_{e}$ & Kinetic Reynolds number \\
\hline$R_{m}$ & Magnetic Reynolds number \\
\hline$S_{\rho}, S_{F}$ & MHD interaction parameters or Stuart numbers \\
\hline$s$ & Slip factor \\
\hline$T$ & Absolute temperature $(\mathrm{K})$ \\
\hline $\boldsymbol{V}, V$ & Fluid velocity vector and magnitude $\left(\mathrm{m} \mathrm{s}^{-1}\right)$ \\
\hline$q$ & Volume $\left(\mathrm{m}^{3}\right)$ \\
\hline$u, v, w$ & Velocity components in Cartesian rectangular coordinates \\
\hline$\alpha$ & Exponent of conductivity power law \\
\hline$\beta_{e}, \beta_{i}$ & Hall parameters (electronic, ionic) \\
\hline$\gamma$ & Isentropic exponent or specific heat ratio $c_{P} / c_{V}$ \\
\hline$\varepsilon$ & Ratio $\rho_{1} / \rho_{2}$ of mass densities \\
\hline$\zeta$ & Ratio $\mathscr{L}_{N} \mathscr{L}_{\rho}$ of length scales for molar mass and mass density \\
\hline$\eta$ & Magnetic parameter \\
\hline$\chi_{1}, \chi_{2}$ & Angles of velocity with respect to shock normal (upstream, downstream) \\
\hline$\chi_{b}$ & Angle of magnetic field with respect to shock normal \\
\hline$\mu_{0}$ & Permittivity of vacuum $\left(\mathrm{H} \mathrm{m}^{-1}\right)$ \\
\hline$\nu_{1}$ & Kinematic viscosity upstream $\left(\mathrm{m}^{2} \mathrm{~s}^{-1}\right)$ \\
\hline$v_{e}, v_{i}$ & Collision frequencies (electrons, ions) $(\mathrm{Hz})$ \\
\hline$\rho$ & Mass density $\left(\mathrm{kg} \mathrm{m}^{-3}\right)$ \\
\hline$\sigma_{e}, \sigma_{P}, \sigma_{H}, \sigma_{C}$ & Electrical conductivities (ordinary, Pedersen, Hall, Cowling) $\left(\mathrm{S} \mathrm{m}^{-1}\right)$ \\
\hline
\end{tabular}

Table 2 Ambient physical conditions

\begin{tabular}{lll}
\hline Parameter & $40 \mathrm{~km}$ & $65 \mathrm{~km}$ \\
\hline Mass density $\rho_{1}\left(\mathrm{~kg} \mathrm{~m}^{-3}\right)$ & $3.85 \times 10^{-3}$ & $1.36 \times 10^{-4}$ \\
Pressure $p_{1}(\mathrm{~Pa})$ & 277 & 9.06 \\
Temperature $T_{1}(\mathrm{~K})$ & 251 & 232 \\
Velocity $V_{1}\left(\mathrm{~m} \mathrm{~s}^{-1}\right)$ & 6900 & 7000 \\
Kinematic viscosity $v_{1}\left(\mathrm{~m}^{2} \mathrm{~s}^{-1}\right)$ & 0.00413 & 0.110 \\
Sound speed $c_{\mathrm{s} 1}\left(\mathrm{~m} \mathrm{~s}^{-1}\right)$ & 317 & 305 \\
Mach number $M_{1}$ & 21.8 & 23.0 \\
Mass density length scale $\mathscr{L}_{\rho}(\mathrm{mm})$ & 0.0130 & 0.359 \\
Normal mass flow rate $m\left(\mathrm{~kg} \mathrm{~m}^{-2} \mathrm{~s}^{-1}\right)$ & 26.6 & 0.952 \\
\hline
\end{tabular}


Table 3 Physical conditions behind the shock without Hall effect $\left(\chi_{1}=60^{\circ}, \chi_{b}=150^{\circ} ; B=1 \mathrm{~T} ; \alpha=2, \zeta=10\right)$

\begin{tabular}{|c|c|c|}
\hline Parameter & $40 \mathrm{~km}$ & $65 \mathrm{~km}$ \\
\hline Mass density $\rho_{2}\left(\mathrm{~kg} \mathrm{~m}^{-3}\right)$ & $3.7496 \times 10^{-2}$ & $6.17 \times 10^{-4}$ \\
\hline Pressure $p_{2}(\mathrm{~Pa})$ & 41614.2 & 1045 \\
\hline Temperature $T_{2}(\mathrm{~K})$ & 3336 & 4403 \\
\hline Isentropic exponent $\gamma_{2}$ & 1.219 & 1.171 \\
\hline Mass density ratio $\varepsilon$ & 0.103 & 0.220 \\
\hline Magnetic parameter $\eta$ & 0.999858 & 0.838 \\
\hline Electrical conductivity $\sigma_{e 2}\left(\mathrm{~S} \mathrm{~m}^{-1}\right)$ & 127.1 & 221 \\
\hline Pedersen current $J_{z 2}\left(\mathrm{kA} \mathrm{m}^{-2}\right)$ & 682 & 1063 \\
\hline Stuart number $S_{\rho}$ & $2.48 \times 10^{-4}$ & 0.333 \\
\hline Stuart number $S_{\rho \sigma}$ & $-3.26 \times 10^{-4}$ & -0.388 \\
\hline Stuart number $S_{v \sigma}$ & $+2.00 \times 10^{-4}$ & +0.292 \\
\hline$a$ & 1.0000814 & 1.0865 \\
\hline$b$ & $-8.678 \times 10^{-5}$ & -0.1126 \\
\hline$c$ & $1.410 \times 10^{-4}$ & 0.1497 \\
\hline$d$ & 0.999850 & 0.8049 \\
\hline$f$ & 0 & 0 \\
\hline$u_{2}\left(\mathrm{~m} \mathrm{~s}^{-1}\right)$ & 355.2 & 773.7 \\
\hline$v_{2}\left(\mathrm{~m} \mathrm{~s}^{-1}\right)$ & 5991.0 & 5097.4 \\
\hline$w_{2}\left(\mathrm{~m} \mathrm{~s}^{-1}\right)$ & 0 & 0 \\
\hline Electric field $E_{x}\left(\mathrm{~V} \mathrm{~m}^{-1}\right)$ & 0 & 0 \\
\hline Electron density $n_{e}\left(\mathrm{~m}^{-3}\right)$ & $1.80 \times 10^{20}$ & $1.04 \times 10^{19}$ \\
\hline Electron density $n_{e}{ }^{*}\left(\mathrm{~m}^{-3}\right)$ & $3.00 \times 10^{17}$ & $7.23 \times 10^{17}$ \\
\hline Electron density $n_{e u}\left(\mathrm{~m}^{-3}\right)$ & $5.78 \times 10^{20}$ & $3.62 \times 10^{19}$ \\
\hline Damköhler number $N_{D \mathrm{~N} 2}$ & 338 & 130 \\
\hline Damköhler number $N_{D O 2}$ & 90.6 & 34.9 \\
\hline Damköhler number $N_{D \mathrm{M} 1}$ & $3.64 \times 10^{-14}$ & $3.08 \times 10^{-11}$ \\
\hline Damköhler number $N_{D \mathrm{M} 2}$ & $3.05 \times 10^{-6}$ & $5.36 \times 10^{-5}$ \\
\hline
\end{tabular}


Table 4 Physical conditions behind the shock with Hall effect $\left(\chi_{1}=60^{\circ}, \chi_{b}=150^{\circ} ; B=1 \mathrm{~T} ; \alpha=2, \zeta=10\right)$

\begin{tabular}{lll}
\hline Parameter & $40 \mathrm{~km}$ & $65 \mathrm{~km}$ \\
\hline Mass density $\rho_{2}\left(\mathrm{~kg} \mathrm{~m}^{-3}\right)$ & $3.7524 \times 10^{-2}$ & $1.49 \times 10^{-3}$ \\
Pressure $p_{2}(\mathrm{~Pa})$ & 41623.8 & 1533 \\
Temperature $T_{2}(\mathrm{~K})$ & 3335 & 2998 \\
Isentropic exponent $\gamma_{2}$ & 1.219 & 1.192 \\
Mass density ratio $\mathcal{E}$ & 0.1026 & 0.0910 \\
Magnetic parameter $\eta$ & 0.999991 & 0.999777 \\
Electrical conductivity $\sigma_{e 2}\left(\mathrm{~S} \mathrm{~m}^{-1}\right)$ & 127.0 & 103 \\
Pedersen current $J_{z 2}\left(\mathrm{kA} \mathrm{m}^{-2}\right)$ & 44.7 & 1.335 \\
Hall current $J_{y 2}\left(\mathrm{kA} \mathrm{m}^{-2}\right)$ & 166.2 & 6.316 \\
Stuart number $S_{\rho}$ & $2.48 \times 10^{-4}$ & 0.154 \\
Stuart number $S_{\rho \sigma}$ & $-3.25 \times 10^{-4}$ & -0.190 \\
Stuart numbers $S_{v \sigma} S_{w \sigma}$ & $+2.00 \times 10^{-4}$ & +0.130 \\
Product $S_{\rho \sigma} S_{w \sigma}$ & $-6.50 \times 10^{-8}$ & -0.0247 \\
$a$ & 1.0000053 & 1.00011 \\
$b$ & $-5.691 \times 10^{-6}$ & $-1.348 \times 10^{-4}$ \\
$c$ & $9.249 \times 10^{-6}$ & $1.965 \times 10^{-4}$ \\
$d$ & 0.99999014 & 0.9998 \\
$f$ & $-2.577 \times 10^{-5}$ & $-6.973 \times 10^{-4}$ \\
$u_{2}\left(\mathrm{~m} \mathrm{~s}^{-1}\right)$ & 354.9 & 319.6 \\
$v_{2}\left(\mathrm{~m} \mathrm{~s}^{-1}\right)$ & 5991.8 & 6081.6 \\
$w_{2}\left(\mathrm{~m} \mathrm{~s} \mathrm{~s}^{-1}\right)$ & -0.154 & -4.241 \\
Electric field $E_{x}\left(\mathrm{~V} \mathrm{~m}^{-1}\right)$ & -755 & -35.5 \\
Electron density $n_{e}\left(\mathrm{~m}^{-3}\right)$ & $1.80 \times 10^{20}$ & $6.66 \times 10^{18}$ \\
Electron density $n_{e}^{*}\left(\mathrm{~m}^{-3}\right)$ & $3.00 \times 10^{17}$ & $6.89 \times 10^{15}$ \\
Electron density $n_{e u}\left(\mathrm{~m}^{-3}\right)$ & $5.77 \times 10^{20}$ & $1.89 \times 10^{19}$ \\
Damköhler number $N_{D \mathrm{~N} 2}$ & 338 & 388 \\
Damköhler number $N_{D \mathrm{D} 2}$ & 29.0 & 31.4 \\
Damköhler number $N_{D \mathrm{M} 1}$ & $3.61 \times 10^{-14}$ & $1.12 \times 10^{-15}$ \\
Damköhler number $N_{D \mathrm{M} 2}$ & $3.04 \times 10^{-6}$ & $5.69 \times 10^{-7}$ \\
Electron Hall parameter $\beta_{e}$ & 4.41 & 96.3 \\
Ion Hall parameter $\beta_{i}$ & $8.67 \times 10^{-3}$ & 0.229 \\
Slip factor $s$ & 0.0382 & 22.1 \\
\hline
\end{tabular}

